\title{
The regulatory genome constrains protein sequence evolution: Implications for the search for disease-associated genes
}

\author{
Patrick Evans ${ }^{\text {Corresp., } 1}{ }^{\text {, Nancy J Cox }}{ }^{1}$, Eric R Gamazon ${ }^{\text {Corresp. 1, 2, 3, } 4}$ \\ 1 Division of Genetic Medicine, Vanderbilt University Medical Center, Nashville, TN, USA \\ 2 Clare Hall, University of Cambridge, Cambridge, United Kingdom \\ 3 MRC Epidemiology Unit, University of Cambridge, Cambridge, United Kingdom \\ 4 Data Science Institute, Vanderbilt University, Nashville, TN, United States \\ Corresponding Authors: Patrick Evans, Eric R Gamazon \\ Email address: patrick.evans@vanderbilt.edu, eric.gamazon@vanderbilt.edu
}

The development of explanatory models of protein sequence evolution has broad implications for our understanding of cellular biology, population history, and disease etiology. Here we analyze the GTEx transcriptome resource to quantify the effect of the transcriptome on protein sequence evolution in a multi-tissue framework. We find substantial variation among the central nervous system tissues in the effect of expression variance on evolutionary rate, with highly variable genes in the cortex showing significantly greater purifying selection than highly variable genes in subcortical regions (Mann-Whitney $\mathrm{U} p=1.4 \times 10^{-4}$ ). The remaining tissues cluster in observed expression correlation with evolutionary rate, enabling evolutionary analysis of genes in diverse physiological systems, including digestive, reproductive, and immune systems. Importantly, the tissue in which a gene attains its maximum expression variance significantly varies $\left(p=5.55 \times 10^{-284}\right)$ with evolutionary rate, suggesting a tissue-anchored model of protein sequence evolution. Using a large-scale reference resource, we show that the tissue-anchored model provides a transcriptome-based approach to predicting the primary affected tissue of developmental disorders. Using gradient boosted regression trees to model evolutionary rate under a range of model parameters, selected features explain up to $62 \%$ of the variation in evolutionary rate and provide additional support for the tissue model. Finally, we investigate several methodological implications, including the importance of evolutionary-rate-aware gene expression imputation models using genetic data for improved search for disease-associated genes in transcriptome-wide association studies. Collectively, this study presents a comprehensive transcriptome-based analysis of a range of factors that may constrain molecular evolution and proposes a novel framework 
for the study of gene function and disease mechanism. 
1 The regulatory genome constrains protein sequence evolution: Implications for 2 the search for disease-associated genes

3

4 Patrick Evans ${ }^{1,}{ }^{*}$, Nancy J. Cox ${ }^{1}$, Eric R. Gamazon ${ }^{1,2,3,4}$ *

5

$6 \quad{ }^{1}$ Division of Genetic Medicine, Vanderbilt University Medical Center, Nashville, TN 37232

$7 \quad{ }^{2}$ Clare Hall, University of Cambridge, Cambridge, CB3 9AL, United Kingdom

$8{ }^{3}$ MRC Epidemiology Unit, University of Cambridge, Cambridge, CB2 0QQ, United Kingdom

$9 \quad{ }^{4}$ Data Science Institute, Vanderbilt University, Nashville, TN 37232

12 Corresponding authors:

13 Patrick Evans, Ph.D. <patrick.evans@vanderbilt.edu>

14 Eric R. Gamazon, Ph.D. <ericgamazon@gmail.com> (Lead Contact)

152215 Garland Avenue 519A Light Hall Nashville, TN 37232

16

17

18 


\section{Abstract}

The development of explanatory models of protein sequence evolution has broad implications

21 for our understanding of cellular biology, population history, and disease etiology. Here we analyze the

GTEx transcriptome resource to quantify the effect of the transcriptome on protein sequence evolution

in a multi-tissue framework. We find substantial variation among the central nervous system tissues in

the effect of expression variance on evolutionary rate, with highly variable genes in the cortex showing significantly greater purifying selection than highly variable genes in subcortical regions (Mann-Whitney

$\left.U \mathrm{p}=1.4 \times 10^{-4}\right)$. The remaining tissues cluster in observed expression correlation with evolutionary rate, enabling evolutionary analysis of genes in diverse physiological systems, including digestive, reproductive, and immune systems. Importantly, the tissue in which a gene attains its maximum expression variance significantly varies $\left(p=5.55 \times 10^{-284}\right)$ with evolutionary rate, suggesting a tissueanchored model of protein sequence evolution. Using a large-scale reference resource, we show that the tissue-anchored model provides a transcriptome-based approach to predicting the primary affected tissue of developmental disorders. Using gradient boosted regression trees to model evolutionary rate under a range of model parameters, selected features explain up to $62 \%$ of the variation in evolutionary rate and provide additional support for the tissue model. Finally, we investigate several methodological implications, including the importance of evolutionary-rate-aware gene expression imputation models using genetic data for improved search for disease-associated genes in transcriptome-wide association studies. Collectively, this study presents a comprehensive transcriptome-based analysis of a range of factors that may constrain molecular evolution and proposes a novel framework for the study of gene function and disease mechanism. 


\section{Introduction}

42
Protein sequence evolution is a central concern for the fields of molecular biology and comparative genomics. Indeed, characterizing the determinants of the rate of protein evolution may help to clarify a wide range of biological processes and phenomena, including the molecular basis of adaptation, the evolution of speciation, and the genetic etiology of disease. Although numerous studies have proposed possible determinants (Drummond et al., 2005; Drummond \& Wilke, 2008; Yang, Zhuang \& Zhang, 2010; Yang et al., 2012), the underlying mechanisms and potential interactions remain unclear. Protein evolution reflects both the rate at which new nucleotide mutations arise and the rate of repair. A second engine of protein evolution, fixation of new mutations depends upon the fitness effect of the relevant mutations and the balance between selection and genetic drift, which is in part mediated by effective population size. We can quantify selection pressures acting on protein-coding regions using the widely used $d N / d S$ ratio. The ratio compares the rate of substitutions ( $K_{a}$ or $\left.d N\right)$ at nonsynonymous sites, which are presumed to undergo selection, to the rate of substitutions ( $\mathrm{K}_{\mathrm{s}}$ or $\mathrm{dS}$ ) at synonymous sites, which are presumed neutral so that dS may serve as a proxy for mutation rate (Kimura, 1990) (although selection may operate on silent sites to favor certain codons (Akashi, 2001; Plotkin \& Kudla, 2011; Quax et al., 2015)). The ratio provides evidence for selective constraint if $d N / d S$ is significantly less than 1 , for neutrality if $d N / d S$ equals 1 , or for positive selection if $d N / d S$ is significantly greater than 1 . Requiring $\mathrm{dN} / \mathrm{dS}$ to be significantly greater than one is generally conservative, as positive evolution generally acts on one region or domain of a protein while the rest of the protein evolves under purifying selection; hence $\mathrm{dN} / \mathrm{dS}$ may potentially overlook positions under positive selection. Here we use the ratio dN/dS as a measure of protein "evolutionary rate," as previously described (Zhang \& Yang, 2015). The rate of protein evolution may vary greatly within and between species (Duret \& Mouchiroud, 2000; Rocha \& Danchin, 2004; Drummond et al., 2005; Larracuente et al., 2008), and elucidating the causes of this variation is an important question in molecular evolution.

Peer] reviewing PDF | (2020:03:46779:1:2:NEW 16 Jun 2020) 
Genetic mutations typically alter phenotype either by altering proteins or by affecting their

regulation. Indeed, differential regulation of gene expression is thought to underlie the remarkable divergence in traits (e.g., behavioral) between humans and chimpanzees especially given the high degree of similarity between orthologous protein sequences (King \& Wilson, 1975). Much of the early work was performed in single cell organisms, such as yeast, raising the question of the generalizability of the results to multicellular eukaryotes (Pál, Papp \& Hurst, 2001; Akashi, 2003; Drummond, Raval \& Wilke, 2006). Different tissues and cell types express different genes and the regulation of gene expression may vary substantially across tissues and cell types, strongly influencing organism-level traits and modulating the evolution of phenotypic novelty. Furthermore, the expression profile of some genes may exhibit substantial developmental-stage specificity.

Molecular evolution in coding sequences has been investigated in many taxa, including humans, and reported to be determined by several (potentially mutually correlated) factors, including expression level, expression breadth, recombination rate, robustness to mistranslation, and connectivity in a biophysical network (Duret \& Mouchiroud, 2000; Pál, Papp \& Hurst, 2001; Fraser et al., 2002; Jordan et al., 2002; Wall et al., 2005; Drummond et al., 2005; Larracuente et al., 2008; Drummond \& Wilke, 2008; Park \& Choi, 2010; Hudson \& Conant, 2011; Shen et al., 2011; Kryuchkova-Mostacci \& Robinson-Rechavi, 2015). Despite the complexity and spatiotemporality of gene expression in a multicellular organism (with its long lifespan and developmental processes), the expression level of a gene has been shown to be a primary predictor of the rate of protein sequence evolution with a variety of possible underlying mechanisms proposed (Akashi, 2003; Rocha \& Danchin, 2004; Lemos, Meiklejohn \& Hartl, 2004;

Drummond et al., 2005). Expression breadth has also been shown to affect protein evolutionary rate, though expression level and breadth have a strong correlation (Duret \& Mouchiroud, 2000; Zhang \& Li, 2004; Liao, Scott \& Zhang, 2006; Park et al., 2012). However, studies of the relationship between gene expression and protein evolution have been limited to divergence data for several hundred to thousand

Peer) reviewing PDF | (2020:03:46779:1:2:NEW 16 Jun 2020) 
89

genes, and to expression data measured in a small number of tissues (Duret \& Mouchiroud, 2000;

Wagner, 2005) using microarrays.

Recent years have witnessed an explosion of large-scale genomic and other types of omics data in a variety of tissue and cellular contexts (Pickrell et al., 2010; GTEx Consortium, 2013; Lappalainen et al., 2013), motivating our attempt at an integrated view of the evolutionary signature of known genes. Here we report a comprehensive transcriptome-based analysis of the factors that may constrain the rate of protein evolution and examine their relevance to sets of genes that define a spectrum of clinical and disease effects, from one end, the essential genes, to the other end, the loss-of-function [LOF] tolerant genes (Ashburner et al., 2000; Blair et al., 2013). Notably, we examine several methodological implications on genomic approaches to mapping disease-relevant genes and on the study of gene function.

\section{Materials \& Methods}

\section{$\underline{\text { Data }}$}

We utilized evolutionary conservation scores from BioMart (Smedley et al., 2015) for humanchimp and human-mouse comparisons. In our analyses, we collected synonymous (dS) and nonsynonymous (dN) substitution rates for 23,816 genes from these comparisons. Proteins vary by two to three orders of magnitude in their rate of evolution (dN/dS).

We utilized GTEx v6p release expression data for 53 tissues involving 8,555 RNA-Seq samples.

The mean RPKM and median RPKM were calculated for each gene in each tissue. The variance and ratio of variance to mean were also calculated for each gene in each tissue to estimate inter-individual transcriptional variability (see sample size for each tissue in Supplementary Table 1). For each gene, we identified the tissue in which the gene shows maximum expression variance. A list of the 53 tissues with 
111 information on sample size is in Supplementary Table 1. In addition to the multi-region sampling of the

112 brain, "frontal cortex" and "cerebellar hemisphere" (obtained after receipt by the brain bank) were

113 sampled by the GTEx Consortium in duplicate ("cortex" and "cerebellum," respectively, as the first

114 replicate obtained at initial tissue collection). Availability of lymphoblastoid cell lines (LCLs, derived from

115 blood) and cultured primary fibroblasts (from skin) provided an opportunity to compare the expression

116 variance and network constraints (see below) in the cell types with those in the tissues of origin.

For all correlation analyses presented, we used log2-transformed RPKM values. We calculated

118 the Spearman's $\rho$ and the corresponding $p$-value with evolutionary rate for all predictors using the R

119 statistical software. To fit a nonlinear model (e.g., of the effect of expression on evolutionary rate), we

120 assumed a generalized additive model using cubic regression spline as "smooth function."

121 To test for the robustness of our results to technical and experimental confounding, we

122 calculated the Probabilistic Estimation of Expression Residuals (PEER) (Stegle et al., 2012) factors (which

123 are based on Factor Analysis) and used the residual to test the correlation of expression level and

124 expression variability with evolutionary rate. We compared results across all tissues (Spearman

125 correlation) between pre-PEER and post-PEER analysis.

126 To quantify tissue expression breadth, we applied the tau $(\tau)$ statistic to the 53 GTEx tissues,

127 with the exception that the frontal cortex was the only brain region used so that the highly correlated

128 tissue samples from the brain would not bias the estimate (Yanai et al., 2005; Kryuchkova-Mostacci \&

129 Robinson-Rechavi, 2016).

130

$$
\tau=\sum_{i=1}^{n} \frac{1-\hat{x}_{i}}{n-1}
$$

131 where 


$$
\hat{x}_{i}=x_{i} / \max _{1 \leq j \leq n}\left\{x_{j}\right\}
$$

133

and $\boldsymbol{x}_{\boldsymbol{i}}$ provides the RPKM value for the gene $\boldsymbol{i}$, and $\boldsymbol{n}$ is the number of tissues. We note that $\tau$ yields a score between zero and one, with zero indicating the same expression across tissues and a value of one indicating strong tissue-specific expression. Genes with similarly high levels or similarly low levels across tissues would have high "expression breadth," which here thus refers to the extent of similarity across tissues. In the actual data, $\tau$ attains a minimum of 0.204 and median of 0.82 for protein-coding genes, underscoring the high tissue specificity of a large set of protein-coding genes. Thus, $\tau$ also reflects intertissue variability (to be distinguished from inter-individual variability in each tissue) in the expression of a gene.

We utilized protein network data in Homo sapiens obtained from STRING v10 (Szklarczyk et al., 2015 ) to investigate the correlation of the number of interactions with evolutionary rate and expression.

We examined several subsets of genes that define a spectrum of phenotypic effects. We obtained and curated a compendium of Mendelian disease genes from the Online Mendelian Inheritance in Man (OMIM). Essential genes were obtained from a study (Georgi, Voight \& Bućan, 2013) that identified human orthologs of mouse genes with known lethal phenotype from the Mouse Genome Database (Blake et al., 2011). LOF-tolerant genes, which can be inactivated without obvious clinical effect, were collected from a comprehensive survey of LOF variants in protein-coding genes (MacArthur et al., 2012). We also investigated immune response genes and olfactory genes, as annotated by the Gene Ontology Consortium (Gene Ontology Consortium, 2015).

\section{Tissue-anchored model of evolutionary rate}

We identified the tissue (MaxTissue) in which a gene attains its maximum inter-individual expression variance (MaxVariance) - and, thus, perhaps the tissue in which the gene exhibits its full 
154 range of functional activity - and tested the MaxTissue's association with evolutionary rate. We should 155 emphasize that the MaxTissue is not necessarily the only tissue in which a gene functions, and indeed a 156 gene may have multiple functions in different tissues. A rejection of the null hypothesis would indicate 157 support for the hypothesis (i.e., the "tissue-anchored model") that when genes are classified into their 158 MaxTissues, the variance $\sigma_{\text {across }}^{2}$ in evolutionary rate across tissues is greater than the variance $\sigma_{\text {within }}^{2}$ 159 of the tissues. tissue or a class of tissues, we calculated the median evolutionary rate and a metric, MaxTissue- $\delta$, for each MaxTissue, defined as

$$
3.14\left(v_{75}-v_{25}\right) / \sqrt{n}
$$

where $v_{75}$ and $v_{25}$ are the $75^{\text {th }}$ and $25^{\text {th }}$ percentile for evolutionary rate respectively and $n$ is the number of genes. We also performed empirical analysis by permutation-based tissue assignment $(\mathrm{N}=1000)$, preserving the gene count in the observed MaxTissue configuration, for the entire protein-coding set with available human-mouse data on rates of nonsynonymous and synonymous substitutions. For each permutation of the entire set (RandomTissue), the $\mathrm{H}$-statistic from the Kruskal-Wallis test was calculated. The empirical p-value was the proportion of the total number of permutations in which the

RandomTissue $\mathrm{H}$-statistic matched or exceeded the observed value from the MaxTissue assignment. evolutionary rate of the genes for which the tissue is a MaxTissue. MaxTissue- $\delta$ is thus a kind of 
175 statistically significant difference between tissues for this evolutionary signature. (We note that this

176 signature is to be distinguished from the median evolutionary rate of all expressed genes in a tissue.)

The tissue-anchored model starts from the observation that the correlation between within-

tissue features (e.g., expression level) and evolutionary rate significantly varies by tissue, raising the

centrality of a cross-tissue analysis. The model would suggest that variation between developmental

programs in which transcription and other within-tissue features exert their effect may be contributing

to variation in evolutionary rate. In each MaxTissue, we ranked the genes according to expression

(Benjamini-Hochberg FDR<0.05) for the top genes $(\mathrm{N}=100)$ in a MaxTissue. The presence of significant,

non-overlapping biological processes between MaxTissues with significant difference in median

constraining evolutionary rate.

\section{Tissue-anchored model: constraint of developmental programs on evolutionary rate}

To further investigate the physiological and developmental mechanisms underlying the tissue-

anchored model of evolutionary rate, we sought to determine to what extent the MaxTissue for a gene could predict the primary affected tissue of developmental disorders associated with the gene. We 
198

200

201

202

204

205

206

207

208

209

210

211

212

213

214

215

216

217

\section{Testing for independent effects given noisy omics data and estimation of spurious correlation}

Partial rank correlation analysis of omics data between two variables, $D$ (a determinant) and $K$

(evolutionary rate), while controlling for a third variable $X$ may generate spurious results (Liu, 1988;

Drummond, Raval \& Wilke, 2006). Consider the case in which a noisy version $X^{\prime}$ is distributed with mean equal to $X$ and variance equal to $\sigma_{X}^{2}$ :

We assume that $D$ and $K$ have the following distributions: $D \sim\left(X, \sigma_{D}^{2}\right)$ and $K \sim\left(X, \sigma_{K}^{2}\right)$. Then the partial rank correlation $r_{D K \mid X}$, between $D$ and $K$ given $X^{\prime}$ simplifies to the following expression:

$$
r_{D K \mid X^{\prime}}={ }^{\sigma_{X^{\prime}}^{2}} / f\left(\sigma_{D^{2}}^{2}, \sigma_{K^{\prime}}^{2}, \sigma_{X^{\prime}}^{2}\right)
$$

Here the denominator is some function of all the variances. Importantly, the numerator is nonzero in the presence of noise in $X$ even under the null hypothesis (i.e., $r_{D K \mid X}=0$ ), leading to a spurious correlation. To determine whether the determinant $D$ contributes to $K$ after controlling for $X$ (given that conventional partial rank correlation analysis generates spurious correlations when applied to noisy biological data), we performed permutation analysis, in which $D$ and $X$ ' were shuffled together (thus preserving their correlation) $n$ times (here $n=1000$ ) and the partial rank correlation between $D$ and $K$ was assessed within each such permutation null set. This generates an empirical p-value for the significance of the observed nonzero correlation coefficient (as the proportion of the permutation null sets that match or exceed the observed correlation coefficient) as well as quantifies the magnitude of the spurious correlation. The permutation null distribution $M_{0}$ for $r_{D K \mid X}$ ' can be used to estimate an adjusted partial rank correlation coefficient: 


$$
\widehat{\vartheta_{D K \mid X}}=\sqrt{\left.r_{D K \mid X}^{2}-\left(E \widehat{(M}_{0}\right)\right)^{2}}
$$

219 where $r_{D K \mid X}$, is the observed partial rank correlation coefficient and $E($.$) is the expectation operator. We$ 220 call this approach Empirical Partial Rank Correlation Analysis (EPRCA), which facilitates a test for

221 independent effects on evolutionary rate. For example, since expression level and tissue breadth were

222 found to be correlated, we tested their independent effect on evolutionary rate using this approach. The

223 approach, in addition, provides an estimate of the extent of the spurious correlation (from the mean

224 and standard deviation of the permutation null distribution).

\section{Correlation between expression and evolutionary rate given co-expression} may bias our estimate of the correlation between gene expression and evolutionary rate. We therefore expression network (shown here, for convenience, for the nonsynonymous substitution rate, but our approach extends, without loss of generality, to $d N / d S)$ :

$$
\operatorname{var}(\varepsilon)=\sigma^{2} \Delta
$$

where $d N$ is the vector of nonsynonymous substitution rates for a set of genes, $G$ is a vector of gene

234 expression, $\pi$ is the effect size, $\Delta$ is the (known) gene expression covariance matrix, and $\sigma^{2}$ is the unknown (absolute) scale. The GLS effect size estimate solves the following minimization problem:

$$
\hat{\pi}=\underset{\pi}{\operatorname{argmin}}\left\{(G-\pi d N)^{T} \Delta^{-1}(G-\pi d N)\right\}
$$

237 which implies: 


$$
\hat{\pi}=\left(d N^{T} \Delta^{-1} d N\right)^{-1} d N^{T} \Delta^{-1} G
$$

$$
\operatorname{var}(\hat{\pi})=\left(d N^{T} \Delta^{-1} d N\right)^{-1} \sigma^{2}
$$

We can view the gene expression traits as mapping to a phylogenetic tree such that the covariance

matrix $\Delta$ captures the covariance between each pair of tips in the tree. The GLS model implements regression that accounts for the phylogeny.

\section{Null phylogenies and null networks}

To assess the significance of the correlation between amino acid substitution rate and

the evolutionary rate estimates for genes while preserving gene branch assignment within the

the observed statistic in the actual data.

\section{Confounding due to tissue diversity sampling} the 44 tissues). 
259

260

261

262

263

264

265

266

267

268

269

270

271

272

273

274

275

276

277

278

279

\section{Cis heritability of gene expression}

We estimated the proportion of gene expression variance captured by local genetic variation and quantified its contribution to evolutionary rate. We considered the following linear mixed model:

$$
Y=W+T+\varepsilon
$$

$$
\operatorname{var}(Y)=A \sigma^{2}+B{\sigma_{T}^{2}}^{2}+I \sigma_{\varepsilon}^{2}
$$

where $Y$ is the residual gene expression phenotype $n$-dimensional vector after adjusting for hidden factors (with $n$ equal to the number of samples in the reference transcriptome dataset), $W$ is the polygenic cis contribution to gene expression for SNPs within $1 \mathrm{Mb}$ of the gene, $A$ is the genetic relatedness matrix estimated from the local polymorphism data (Yang et al., 2010), $T$ is the polygenic trans contribution from the remaining common variants (MAF>0.05) in the genome, and $B$ is the genetic relatedness matrix estimated from these trans-variants on the other chromosomes. The variance of the polygenic cis burden $W$ is $A \sigma^{2}$ while that of the polygenic trans burden is $B{\sigma_{T}}^{2}$; the remaining variance attributable to environmental regulation is $I \sigma_{\varepsilon}^{2}$. We estimated these variances using restricted maximum likelihood, as implemented in GCTA (Yang et al., 2011), allowing us to quantify the SNP-based cis heritability of gene expression as $h_{Y}^{2}=\sigma^{2} /\left(\sigma^{2}+{\sigma_{T}}^{2}+\sigma_{\varepsilon}^{2}\right)$. We used DGN whole blood samples (N=922) for maximal power.

\section{Gradient boosted regression for modeling of evolutionary rate}

Selection of informative features among many predictors and incorporation of possibly nonlinear effects into a functional form for evolutionary rate may provide insights into potential causal factors and their relative contributions. The task is a variable selection and model choice problem. Although prediction is not our primary aim in this context, a modeling approach that is robust to 
280

281

282

284

285

286

287

288

289

290

291

292

293

294

295

296

297

298

299

300

overfitting and to redundancy (or multicollinearity) is desired. We therefore modeled $\mathrm{dN} / \mathrm{dS}$, based on the human-mouse comparison, using gradient boosted regression trees. The approach combines, in an iterative fashion, otherwise "weak" models or classifiers into a "strong" learner. Using a loss function $L(y, F(x))$ on the evolutionary rate $y$ and the model $F(x)$ built on a vector $x$ of features, the approach seeks to incrementally boost the prediction:

$$
\begin{gathered}
M_{0}(x)=\operatorname{argmin}_{\alpha} \sum_{i=1}^{n} L\left(y_{i}, \alpha\right) \\
M_{p}(x)=M_{p-1}(x)+\operatorname{argmin}_{h} \sum_{i=1}^{n} L\left(y_{i}, M_{p-1}\left(x_{i}\right)+h\left(x_{i}\right)\right)
\end{gathered}
$$

Here $x_{i}$ is a vector of features for $y_{i}, n$ is the number of training set observations, $\alpha$ is a constant, $p$ is the iteration index, $M_{p}$ is the $p$-th model, and $h$ is a base learner (tree) fitted to improve on the model $M_{p-1}$ . (The residual $y_{i}-F\left(x_{i}\right)$ can be interpreted as a negative gradient.)

The models were fitted using the "gbm" R package with a squared error loss function. We utilized a range of values for model parameters. We fitted $N_{t}(100,500,1000,10000)$ trees with interaction depth of $D_{i}(1,4,5)$, indicating a model with up to $D_{i}$-way interactions (which limits the number of nodes in a tree), and a shrinkage parameter of 0.01 (which shrinks the contribution of each tree). We also performed 10 -fold cross validation to evaluate the generalizability of the model. Each combination of model parameters provides a "feature importance score", generating a predictor importance spectrum that shows the relative importance of each input feature in predicting evolutionary rate. The feature importance score provides support for how MaxTissue is defined in the proposed tissue-anchored model. In addition, using 100 and 500 iterations, $N_{t}=1000, D_{i}=4$, and shrinkage parameter of 0.01 , we trained on $70 \%$ of the data to predict the complement set and to obtain a distribution of out-of-sample adjusted $R^{2}$. 
301

302

303

304

305

306

307

308

309

310

311

312

313

314

315

316

317

318

319

\section{0}

321

322

\section{Gene expression imputation using PrediXcan}

PrediXcan is an approach for estimating the genetically determined component of gene expression (Gamazon et al., 2015a) using only the germline genetic profile; this estimate is then tested for association with disease risk. Since the disease trait is not likely to modify the germline genetic profile, an observed association comes with a proposed causal direction. Thus, even when directly measured expression is available, its genetic component, though likely to be correlated with the total expression level, provides additional mechanism-relevant information not influenced by disease. Gene expression imputation models were generated using the GTEx reference transcriptome panel in 44 tissues (each with at least 70 samples), as previously described (Gamazon et al., 2015a). Each gene expression model (consisting of selected SNPs within $1 \mathrm{MB}$ of the given gene and their additive

regulatory effect size on expression) comes with a measure of imputation performance, namely, the 10fold cross-validation $R^{2}$ (the square of the correlation between imputed and observed expression) estimated within each tissue (from GTEx or DGN). We note that the best eQTL for a gene also provides an example of an imputation model, though possibly suboptimal in predictive performance. Thus, the practice of testing such an eQTL for its association with a trait is a specific instance, if suboptimal, of the PrediXcan framework. In contrast, our study utilized multi-SNP imputation models for improved performance. The imputation $R^{2}$ also provides an estimate of the aggregate effect of local genetic variation on gene expression and can be evaluated, in each tissue, for its correlation with evolutionary rate.

\section{Methodological implications}

We assessed several methodological implications on the search for disease-associated genes using both PrediXcan and conventional GWAS. We explored three scenarios. 
323 1. Extent of local genetic control or cis heritability of gene expression: Cis heritability provides an upper

324 bound on how well local genetic variation may be utilized to impute gene expression. We calculated 325 the Spearman correlation between the imputation $R^{2}$ (derived from 10-fold cross validation for each 326 tissue within the PrediXcan framework (Gamazon et al., 2015a)) and evolutionary rate to evaluate to what extent the genetically determined component of the expression of conserved genes, relative to the complement set, may be reliably imputed. We estimated the reduction in statistical power to detect disease associations for conserved genes $(\mathrm{dN} / \mathrm{dS}<0.01)$ relative to fast-evolving genes $(\mathrm{dN} / \mathrm{dS}>1)$ in transcriptome-wide association studies, assuming a modestly sized GWAS $(\mathrm{N}=1000)$ and using the empirical distribution of $R^{2}$ for the gene sets in a typical tissue (skeletal muscle, chosen for its sample size). The significance was assessed using the Mann-Whitney $U$ test.

2. Relevance of cross-tissue imputation models: We assessed the utility of cross-tissue (versus singletissue) predictors by evaluating the impact of expression tissue specificity $(\tau)$ on gene expression imputation. Genes with $\tau$ close to zero have equal expression levels across all tissues, and in this case, tissue-dependent imputation models are likely to suffer from degraded imputation performance; on the other hand, tissue-specific expression profiles would require tissue-dependent imputation models. We tested for enrichment of broadly expressed genes (defined at multiple thresholds, $\tau<0.30,0.40$, and 0.50 ) among conserved genes using random sampling from the set have been shown to be enriched for cis eQTLs (Nicolae et al., 2010; GTEx Consortium, 2015). Indeed, incorporation of eQTL information into GWAS analysis has been shown to improve (quite substantially, for some traits and tissues) the false discovery rate. We calculated the Spearman 
gene) and evolutionary rate. A strong positive correlation would suggest, for instance, that conserved genes may be less likely to be detected as eGenes. This observation would have important implications on the task of attribution of gene mechanisms to GWAS loci. We calculated the same correlation for the set of trait-associated SNPs $\left(p<5 \times 10^{-8}\right)$ found in the NHGRI GWAS catalog that are in linkage disequilibrium $\left(r^{2}>0.80\right)$ with a best eQTL using the eQTL's effect size.

Functional enrichment analysis the lowest $10 \%$ of $\tau$ (i.e., high expression breadth) as well as genes that pass a more stringent threshold (i.e., the 100 most widely expressed genes) to find significantly enriched functional annotations and known pathways for tightly regulated genes. At the other end of the distribution, the 100 genes with the most tissue-specific expression profile were evaluated to identify enriched functional annotations.

Similarly, we analyzed genes with 1 to 10 interactions to characterize the genes that map to the oldest branch of the phylogeny with unexpectedly low connectivity. We also tested the 100 genes at the other end of the node degree distribution to characterize the genes that occupy central network positions.

\section{Results}

We utilized RNA-seq data derived from 53 human tissues to estimate the mean and variance of expression patterns for each gene (the number of samples for each tissue can be found in

To assess evolutionary conservation, for each orthologous gene we estimated $\mathrm{dN} / \mathrm{dS}$ rates by comparing 
369 human-mouse and human-chimp divergence comparisons (Figure 1), as the human-chimp comparison

370 may be more relevant to the evolution of genes involved in human diseases (discussed later) while the

371 human-mouse comparison is less sensitive to small numbers of mutations.

The rate of nonsynonymous substitution ( $\mathrm{dN}$ ) based on the human-chimp comparison shows a

373 significantly negative correlation (Spearman's $\rho$ ranges from -0.32 to $-0.17, p<1.41 \times 10^{-110}$ ) with mean

374 expression level in all 53 tissues. Whenever we present a range for a test statistic, such as Spearman's $\rho$,

375 for all tissues, the p-value presented is always the highest or least significant. The same observation

376 holds (Spearman's $\rho$ ranges from -0.32 to $-0.15, p<1.35 \times 10^{-77}$ ) for evolutionary rate, $d N / d S$. Stronger

377 correlations with similar trends across tissues are obtained from the human-mouse comparison

378 (Spearman's $\rho$ ranges from -0.48 to $-0.24, \mathrm{p}<1.37 \times 10^{-235}$ for $\mathrm{dN}$ and expression level, and Spearman's $\rho$

379 ranges from -0.42 to $-0.19, \mathrm{p}<1.35 \times 10^{-148}$ for $\mathrm{dN} / \mathrm{dS}$ and expression level).

The uniform analytic pipeline applied to the breadth of tissues (from a large number of the same

in a tissue and support for either purifying selection or positive selection. For each gene in a given tissue, we calculated the mean (to quantify level) and variance or ratio of variance to mean (to quantify interindividual variability) of gene expression. Notably, the observed expression correlation with sequence divergence varies considerably across tissues and displays tissue clustering (Figure 1A; human chimp divergence). The brain regions (as previously noted (Duret \& Mouchiroud, 2000)) and, interestingly, cervical spinal cord show the largest absolute magnitude of correlation of expression with evolutionary comparisons. The clustering for the remaining tissues enables comparisons of the contribution of physiological systems, including reproductive, immune, and gastrointestinal systems, to sequence 
392 gastrointestinal systems and reproductive tissues do not. After the neural tissues, the alimentary canal -

393 in particular, the muscularis mucosae of the esophagus, gastro-esophageal junction, and sigmoid colon -

394 has among the largest absolute effects of expression in primary cells on nonsynonymous substitution

395 rate in both divergence datasets.

We examined the tissue distribution of the correlation of expression with silent substitution rate. Selection on synonymous sites has been previously noted in other taxa (Rocha \& Danchin, 2004; Larracuente et al., 2008; Zhou et al., 2016). A negative correlation is observed for dS in most tissues $\mathrm{dN}$ and dS (Spearman's $\rho=0.415, p<1 \times 10^{-300}$ ) based on human-chimp divergence. However, this relationship may merely be due to selective constraints on amino acid changes. Previous studies have indeed attributed the correlation between $\mathrm{dN}$ and $\mathrm{dS}$ to neighboring effects (Duret \& Mouchiroud, 2000), and suggested that neighboring sites may influence a site's mutation rate (Aggarwala \& Voight, 2016). Interestingly, the tissue patterns of the correlation of gene expression with synonymous divergence differ from that with non-synonymous divergence. For example, the highest absolute correlation with dS, based on human-chimp divergence, is in fibroblasts, lymphoblastoid cell lines (LCLs), and skeletal muscle (and not in any of the brain regions); in contrast, the cortex now shows the lowest absolute correlation (Supplementary Table 2). The tissue dependence of the variation of silent substitution rate with expression becomes more significant when using human-mouse divergence, with aorta, sigmoid colon, and tibial nerve (for example) displaying significantly higher absolute effects than

411 the brain regions (Figure 1A). Collectively, these results support the notion that silent sites may be to 412 some degree under selective constraints.

We assessed the contribution of inter-individual transcriptional variability within each tissue, 
415 mean and variance), to evolutionary rate. Expression variance explains similar levels of variation in

416 evolutionary rate as expression mean in all tissues (Figure 1B), as expected from the correlation

417 between expression mean and variance. Highly significant results on the effect of expression variance in each tissue on nonsynonymous substitution rate and evolutionary rate are obtained with human-mouse divergence (Spearman's $\rho$ ranges from -0.46 to $-0.18, \mathrm{p}<2.20 \times 10^{-126}$ for $\mathrm{dN}$ and expression variance, and Spearman's $\rho$ ranges from -0.42 to $-0.15, p<5.20 \times 10^{-90}$ for $\mathrm{dN} / \mathrm{dS}$ and expression variance). Using the ratio of variance to mean to quantify variability, we continue to observe a significant effect on evolutionary rate in each tissue (Spearman's $\rho$ ranging from -0.39 to $-0.08, p<3.49 \times 10^{-26}$ ). unmeasured confounders in gene expression measurements, including any population structure, may bias our analyses. We calculated the Probabilistic Estimation of Expression Residuals (PEER) factors (Stegle et al., 2012) and utilized the residual (thereby reducing the impact of technical artefacts, population structure, and other hidden confounders that may be present in the measurement of gene expression) for downstream analysis. We continued to observe a significant correlation between variance (using residual expression after PEER adjustment) and evolutionary rate in each tissue (Figure 1C), with results between the pre-PEER and the PEER-adjusted analysis being significantly correlated 431 (Spearman's $\rho=0.997)$. the largest absolute effects of within-tissue expression variability on evolutionary rate using either variance or the ratio of variance to mean (Supplementary Table 2 and Figure 1A), but there is also significant variation in observed effects across the CNS tissues. Based on the human-mouse comparison, highly variable genes (defined as the top 1000 in expression variance) in the cortex are under

437 significantly greater purifying selection than highly variable genes in the subcortical regions (Wilcoxon 
438 rank sum $\left.p=1.4 \times 10^{-4}\right)$. On the other hand, highly variable genes in the cervical spinal cord evolve more

439 rapidly than highly variable genes in the brain regions (Wilcoxon rank sum range from $p=5.17 \times 10^{-9}$ for

440 anterior cingulate cortex BA24 to $p=0.007$ for substantia nigra). Whole blood and LCLs tend to show

441 lower contribution of expression variance to variation in evolutionary rate than solid tissues, suggesting

442 that evolutionary analysis in these relatively accessible tissues may not accurately reflect the

443 transcriptome-wide contribution to molecular evolution. Although cell type heterogeneity and Epstein-

444 Barr virus transformation may globally alter gene expression profiles in whole blood and LCLs

445 respectively, we note that spleen, a key component of the immune system, also displays among the

446 lowest absolute effects of expression variance on evolutionary rate (Spearman's $\rho=-0.217, p=3.45 \times 10^{-187}$

447 compared to Spearman's $\rho=-0.419$ for anterior cingulate cortex). Of interest to pharmacogenomics

448 studies, highly variable genes in the liver, a key tissue for drug metabolism and transport (Chhibber et

449 al., 2017), tend to show relatively accelerated evolution (Wilcoxon rank sum $p=2.33 \times 10^{-15}$ for the

450 comparison of muscle and liver), consistent with the hypothesis that pharmacogenes have evolved as a

451 defense mechanism against the accumulation of harmful xenobiotics.

\section{A Gene's MaxTissue is Significantly Associated with Rate of Protein Sequence Evolution}

We find that the tissue ("MaxTissue") in which a gene attains its maximum inter-individual

variance ("MaxVariance") - and perhaps also the tissue in which the gene shows the full range of its

functional activity - is significantly associated with evolutionary rate (human-mouse comparison,

Kruskal-Wallis test $\left.p=5.55 \times 10^{-284}\right)$. (We note that tissue-specific $(\tau>0.95)$ essential genes demonstrate

$p=1.96 \times 10^{-60}$ in whole blood), supporting the potential value of considering the tissues of high or

maximal variance.) The significantly greater variation in evolutionary rate across MaxTissues (than 
461 physiological process in which transcription takes place may constrain protein sequence evolution. To

462 illustrate, among 3 selected MaxTissues (cortex, liver, and testis) with significant variance ( $p=5.55 \times 10^{-284}$ )

463 in median dN/dS of the genes in a MaxTissue, the 100 genes with the highest expression variance from

464 each MaxTissue are significantly enriched (Benjamini-Hochberg FDR<0.10) for non-overlapping sets of

465 functional annotations (see Methods and Figure 2A). In cortex, dendrite, cell junction, and neuron

466 projections are among the functional categories implicated $\left(p=4.6 \times 10^{-3}\right)$; in liver: blood microparticle,

467 complement and coagulation cascades, and metabolism of xenobiotics by cytochrome $P 450\left(p=3.7 \times 10^{-}\right.$

$468{ }^{10}$ ); in testis: spermatogenesis, chromosome condensation, and multicellular organism development

$469\left(p=5.74 \times 10^{-4}\right)$. The non-overlapping functional categories and the significant difference $\left(p=5.55 \times 10^{-284}\right)$ in

470 median evolutionary rate between MaxTissues suggest that variation in the conservation of certain

471 MaxTissue-specific biological and developmental processes significantly contributes to the variation in

472 the rate of protein evolution. Furthermore, permutation analysis that preserves the gene count in the

473 observed MaxTissue configuration finds no random assignment of gene to tissue (RandomTissue;

$474 \mathrm{~N}=1000$ datasets) of the entire protein-coding set that generates a (Kruskal-Wallis) statistic as extreme

475 (see Methods). As expected, the genes with the highest expression variance in RandomTissues show no

476 enriched functional categories. The association is not driven by a single tissue or class of tissues. Using

477 the MaxTissue- $\delta$ metric (see Methods) to estimate the variability in evolutionary rate and to rank the

478 MaxTissues, the cortex (0.075), esophagus (0.061), and vagina (0.061) show among the highest values

479 while fibroblasts (0.013), skeletal muscle (0.014), and whole blood (0.015) among the lowest. The other

480 brain regions are spread throughout (e.g. anterior cingulate cortex (0.016), hippocampus (0.017), and

481 caudate basal ganglia (0.051)). Removing the cortex as MaxTissue, the observed association remains

482 significant $\left(p=1.04 \times 10^{-284}\right)$.

483 A Gene's MaxTissue Predicts Primary Affected Tissue for Developmental Disorders Associated with the

484 Gene

Peer) reviewing PDF | (2020:03:46779:1:2:NEW 16 Jun 2020) 

constrain protein sequence evolution, as proposed by the tissue-anchored model, we tested the extent

487 to which the MaxTissue for a gene could predict the primary affected tissue for developmental disorders associated with the gene using a large-scale, independently curated resource (see Methods). We found that MaxTissue significantly overlapped $\left(p=3.5 \times 10^{-41}\right)$ with the primary affected tissue (Fisher's exact test odds ratio $=4.62$ ), suggesting a potential mechanism for the tissue-anchored model since developmental disorders are likely to affect fitness and therefore the evolutionary rate of the protein. evolutionary rate. We used the tau $(\tau)$ statistic to capture the level of similarity of expression across tissues (i.e., expression breadth) in GTEx (Yanai et al., 2005; Kryuchkova-Mostacci \& Robinson-Rechavi, 2016) (see Methods). In the distribution of $\tau$ by gene type (Supplementary Figure 1), protein-coding genes display the widest variation; in contrast, lincRNAs show substantial tissue specificity in expression compared to protein-coding genes (Supplementary Figure 2). Importantly, using tissue downsampling (see Methods), we find that less comprehensive tissue catalogs than presented here can have substantial variability in the estimated tissue specificity of a gene expression trait (Figure 2B), with significantly higher variance in $\tau$ among genes with higher $\tau$ (Spearman's $\rho=0.359, p<1.0 \times 10^{-300}$ ). $\mathrm{dN} / \mathrm{dS}$ and $\tau$ (Spearman's $\rho=0.25, \mathrm{p}=7.5 \times 10^{-243}$ ) from the human-mouse comparison, consistent with the notion that intensity of selection on nonsynonymous sites is strongly determined by how broadly expressed the gene is (Duret \& Mouchiroud, 2000). genes may indicate functionally relevant differences between regulatory genes and their targets and 
508 enrichment analysis of the lowest $10 \%$ of $\tau$ scores (i.e., genes with the broadest expression profile)

509 implicates functional annotations relating to transcription (Benjamini-Hochberg adjusted $\mathrm{p}=6.19 \times 10^{-36}$ )

510 and mRNA processing (Benjamini-Hochberg adjusted $p=1.57 \times 10^{-34}$ ) (Huang, Sherman \& Lempicki, 2009).

511 More stringently, the 100 genes with the greatest expression breadth are enriched in Ubl conjugation

512 pathway $\left(n=13\right.$, Benjamini-Hochberg adjusted $\left.p=3.7 \times 10^{-3}\right)$, a highly conserved eukaryotic gene

513 regulatory mechanism that frequently promotes protein-protein interactions (Hochstrasser, 2009). Thus,

514 a possible explanation for the importance of expression breadth in constraining evolutionary rate is the

515 conservation of genes involved in multi-tissue gene regulation. In contrast, the 100 genes with the most

516 tissue-specific expression profile are enriched for secreted proteins ( $n=31$, Benjamini-Hochberg adjusted

$\left.517 p=8.5 \times 10^{-9}\right)$ with gene products, for instance, located in extracellular region or involved in signaling.

518 Comparing the two cross-tissue features, we find that $\tau$ explains only $0.88 \%\left(p=6.0 \times 10^{-40}\right)$ of the

519 variability in MaxVariance. Using EPRCA, we find that evolutionary rate and MaxVariance are

520 significantly correlated after controlling for expression breadth (Spearman's $\rho=-0.151$, permutation

$521 \mathrm{p}<0.001)$. These results suggest that expression breadth and MaxVariance, as predictors of between-

522 tissue effects, may independently constrain evolutionary rate.

Joint Feature Analysis of Rate of Protein Sequence Evolution (dN/dS)

Besides MaxTissue and MaxVariance (above), we identified additional correlates of dN/dS.

527 be predicted, to a degree measured by heritability and in a tissue-dependent manner, based on genetic 
531 Although gene age and PPI node degree have been previously investigated (and although the method

532 for gene age estimation may generate methodological artifacts (Moyers \& Zhang, 2017)), we report

533 their relationship with the unique expression data analyzed here (controlling for potential confounders)

534 and find these variables to be significant associated with $\mathrm{dN} / \mathrm{dS}$ (Supplementary Information).

535 The features we have examined are mutually correlated (Figure 3A), posing a challenge to the

536 search for causal factors. A comparison of the generalized additive models based on the human-mouse

537 comparison using AIC (which is asymptotically equivalent to leave-one-out cross-validation for ordinary

538 linear regression (Stone)), is shown in Supplementary Table 4. Notably, tissue expression breadth is the

539 model with the best univariate fit.

We then implemented an approach that performs variable selection and model choice (given

541

542

543

544

545

546

547

548

549

550

551

552

the number of correlated predictors) and incorporates potentially nonlinear effects (given the univariate observation on the presence of such effects). We utilized gradient boosted regression trees to model evolutionary rate (see Methods), based on human-mouse divergence, jointly analyzing all the features.

Notably, we find that MaxVariance, among all features, has the highest feature importance score using a range of model parameters for the number of trees and the number of interactions (see Methods)

(Figure 3B), providing further support to the proper centrality of this feature in our tissue-anchored model of evolutionary rate. In particular, MaxVariance has a stronger influence on predicting evolutionary rate than maximum expression level. We find that the combined model explains an average (and median) of $62 \%$ of the variability in evolutionary rate (Figure $4 \mathrm{~A}$ ) based on out-of-sample prediction (see Methods), with MaxVariance consistently having a higher relative importance than maximum expression level (Figure 4B). The relative importance of MaxVariance in the gradient boosted model is consistent with the single-model finding that a significant association with evolutionary rate 
553 characterizes the MaxTissue configuration and is not observed for a random tissue reconfiguration

554 (permutation $\mathrm{p}<0.001$; see Methods).

555 We also considered specific gene sets to determine to what extent the observed relationships

556 between evolutionary rate and expression features apply (Supplementary Information). Mendelian

557 disease genes and essential genes display substantially lower MaxVariance effect, in terms of variability

558 explained, on evolutionary rate than randomly drawn sets $(\mathrm{N}=1000)$ of proteins, indicating that

559 MaxVariance and gene function may interact to influence evolutionary rate (Supplementary

560 Information and Figure 4C). As in the full set of protein-coding genes, when restricted to Mendelian

561 disease genes for all model assumptions examined, gradient boosted modeling analyses indicate that

562 MaxVariance continues to have a higher relative importance than maximum expression level.

563 Methodological Implications on Genomic Studies of Disease

564 Our collective findings have important methodological implications for the search for disease-

565 associated genes and the study of gene function. We explore three specific applications (using the

566 human-chimp comparison for illustration).

567 The genetically determined component of gene expression in a tissue (see Methods) is typically

568 tested for association with disease in transcriptome-wide association studies (e.g., using PrediXcan

569 (Gamazon et al., 2015a)). Our study would suggest that imputation of genetically determined expression

570 for conserved genes using genetic variants within the cis region of a gene (1 Mb) should be less accurate

571 than for fast-evolving genes. Genes with less accurate expression imputation would suffer from lower

572 statistical power in an association analysis. We note that even if directly measured gene expression data

573 are available, estimation and inference on the genetically determined expression should yield additional

574 information on underlying disease mechanisms.

Peer) reviewing PDF | (2020:03:46779:1:2:NEW 16 Jun 2020) 

out-of-sample imputation $R^{2}$ for gene expression (see Methods) and dN/dS (Spearman's $\rho=0.09$, $577 \mathrm{p}=8.6 \times 10^{-14}$ for adipose subcutaneous; Spearman's $\rho=0.057, p=2.6 \times 10^{-6}$ for skeletal muscle; and 578 Supplementary Table 6 for the remaining tissues). For most tissues, imputation performance is 579 significantly lower for conserved genes than for fast-evolving genes (Figure 5A). The differential 580 performance has important implications for mapping disease-associated genes. Indeed, in a tissue 581 (skeletal muscle, chosen for its sample size) with significant differential imputation performance 582 (Bonferroni-adjusted $p<0.05$ for number of tissues tested) and a modestly sized genome-wide association study ( $\mathrm{N}=1000)$, we estimate that conserved genes would have significantly lower statistical power than fast-evolving genes (median power 0.16 versus 0.41 , Mann-Whitney $U$ test $p=2.2 \times 10^{-6}$; see Methods). Furthermore, a smaller proportion of conserved genes ( 0.32 versus 0.41 ) would have at least $80 \%$ power (Figure 5B). Finally, the significant correlation with out-of-sample imputation $R^{2}$ confirms the significant positive correlation between evolutionary rate and cis heritability, for which estimation, using linear mixed models, depends on a rather strong assumption of polygenicity of gene expression. involved in multi-tissue gene regulation are notable for the relative strength of purifying selection acting on them, contributing to the strong correlation between expression breadth and evolutionary rate. Our study would suggest that imputation of gene expression for conserved genes could be substantially improved using multi-tissue (versus tissue-specific) genetic predictors. Consider the hypothetical case 594 where a gene is broadly expressed and, indeed, has equal expression across all tissues (i.e., $\tau=0$ ). Clearly, an imputation model that varies by tissue would be inconsistent with the gene's true expression profile and, thus, have suboptimal imputation performance. On the other hand, a gene with highly

597 tissue-specific expression profile (e.g., $\tau=1$ ) would require tissue-dependent models for optimal 598 imputation. 

$d N / d S<0.01, N=2,750)$, a significant enrichment $(p<0.001$ ) for broadly expressed genes can be found (see Methods). In general, $4.41 \%$ of the variance in evolutionary rate is explained by expression breadth.

These genes that are subject to the strongest purifying selection show a significant $15 \%$ decrease in tissue specificity $(\tau)$ (Wilcoxon rank sum $p=9.57 \times 10^{-78}$ ) relative to the remaining protein-coding genes.

For a final application, we investigated whether our findings would also yield methodological insights into traditional single-variant analyses of complex traits. Since trait-associated SNPs identified by GWAS have been shown to be enriched for cis eQTLs, we asked where the target genes of these regulatory variants lie in the overall distribution of evolutionary rate. We calculated the Spearman correlation between the best cis eQTL effect (within $1 \mathrm{Mb}$ of target gene) in absolute value and evolutionary rate. We find a significant positive correlation (Spearman's $\rho=0.204$ between absolute cis effect size and $\left.d N / d S, p=1.3 \times 10^{-133}\right)$, suggesting that conserved genes may be less likely to be detected as eGenes. Furthermore, using reproducible trait-associated variants $\left(p<5 \times 10^{-8}\right)$ curated in the NHGRI GWAS catalog that are in linkage disequilibrium $\left(r^{2}>0.80\right)$ with a best eQTL, this correlation becomes raises the possibility that attribution of gene mechanisms to GWAS loci using only cis eQTLs has underestimated the influence of this class of genes.

\section{Discussion}


622 human disease-associated genes, and explored a number of methodological implications for

623 transcriptome-wide association studies (Gamazon et al., 2015b; Gusev et al., 2016). We also present a

624 tissue-anchored model, with MaxTissue a significant tissue configuration for evolutionary rate, and a

625 combined feature set that explains a large proportion of the variation.

626 The greatest effects of expression level, in terms of variance explained, on evolutionary rate are

627 observed for the CNS, including the brain, consistent with an earlier report (Duret \& Mouchiroud, 2000;

628 Gu \& Su, 2007; Tuller, Kupiec \& Ruppin, 2008; Kryuchkova-Mostacci \& Robinson-Rechavi, 2015), and

629 cervical spinal cord. However, we also find a remarkably similar level of contribution from expression

630 variance in the CNS tissues, which remains significant after controlling for the mean level. The sampling

631 of diverse CNS tissues enabled us to observe significant variation in expression constraints on sequence

632 divergence. Notably, highly variable genes in the cortex are under significantly greater purifying

633 selection than highly variable genes in the subcortical regions, illustrating differences in evolutionary

634 conservation for this class of genes even among related neural tissues. The higher absolute effect of

635 expression level or variance on evolutionary rate observed in the CNS system than in other systems may

636 be in part attributable to increased selection to prevent toxicity from protein misfolding and aggregation

637 of highly expressed genes.

A uniform analytic pipeline applied to the diversity of tissues provides a unique opportunity to

estimate the contribution of transcriptional variance in each tissue to evolutionary rate. We tested both

variance and ratio of variance to mean and observe similar tissue-dependent patterns. Several

641 mechanistic hypotheses, for the role of expression level in constraining evolutionary rate, have been

642 proposed (Akashi, 2003; Rocha \& Danchin, 2004; Lemos, Meiklejohn \& Hartl, 2004; Drummond et al.,

643 2005). On the other hand, expression variance captures the range of expression level over which a gene

644 functions in a given tissue and thus potentially reflects a temporal component to expression or a cellular 
645 or developmental process. Instead of maintaining a steady-state value near the mean, some conserved

646 genes may express at low levels and then quickly express to high levels when activated. Rapid cycling of

647 expression of such genes in a tissue may also therefore play a role for the protein folding accounts

648 previously proposed for expression level. In contrast with these within-tissue predictors of evolutionary

649 rate, the association of tissue (MaxTissue) with maximum expression variance with evolutionary rate,

650 which our empirical analysis shows to be a significant tissue configuration, highlights the importance of

651 cross-tissue predictors and the developmental or physiological component of the variation in

652 evolutionary rate.

653

Here, we measure expression breadth in tissues derived primarily from adults (GTEx

654

655

656

657

658

659

660

661

662

663

664

665

666

667
Consortium, 2015), which may underestimate the true expression breadth of genes that have a strong temporal component to their expression. Importantly, tissue downsampling shows that expression breadth for genes expressed in a large number of tissues is robust to tissue sampling but, for genes expressed in a limited set of tissues, is highly sensitive to the choice of tissues. This vulnerability of tissue-specific genes to mismeasurement underscores the utility of a large-scale resource in assessing the importance of tissue breadth, as previous studies of protein sequence evolution have examined only a small number of tissues. This has further implications for studies of younger genes (e.g. primatespecific), which tend to have more tissue-specific expression. In a comparison of cross-tissue predictors (versus within-tissue expression features), expression breadth accounts for only a small proportion (8.9\%) of the variability in MaxVariance, which may therefore represent independent constraint and, indeed, shows the greatest contribution to predicting evolutionary rate among all features.

We find, using whole blood transcriptome data, that cis heritability of gene expression is significantly positively correlated with nonsynonymous substitution rate as well as with evolutionary rate. Genes with low cis heritability of expression tend to be conserved. However, the linear mixed

Peer) reviewing PDF | (2020:03:46779:1:2:NEW 16 Jun 2020) 
668 model approach to SNP-based heritability estimation assumes a polygenic cis architecture of gene

669 expression; this approach may therefore perform poorly in the presence of large-effect regulatory

670 variants. Nevertheless, the out-of-sample imputation $R^{2}$ (which provides an estimate of the cis

671 heritability and should be more robust to model misspecification) from the gene expression (PrediXcan)

672 model also varies with evolutionary rate. One explanation for the observed relationship between

673 heritability and evolutionary rate is that conserved genes have less standing genetic variation in cis in a

674 population, and therefore, lower heritability. However, the estimated heritability does not include the

675 effects of more complex forms of genetic variation or of rare regulatory variation. The tissue variation in

676 the correlation of the imputation $R^{2}$ (as an estimate of heritability) with evolutionary rate suggests that

677 the implied effect of the regulatory genome on protein sequence evolution may in part arise from

678 genetic control of tissue-specific biological processes. For example, genes that underlie specific

679 developmental programs may evolve significantly more slowly than those for other developmental

680 programs, contributing to variation in evolutionary rate.

681

Notably, the correlation of evolutionary rate with gene expression heritability and with

682

683

684

685

686

687

688

689

690

691

proposes that highly expressed genes are conserved due to selection against protein misfolding caused

by nonoptimal amino acids. Importantly, the translational robustness hypothesis leaves unanswered the question of why the correlation between expression and evolutionary rate should, as reported here, significantly vary by tissue. Our proposed tissue-anchored model highlights the centrality of tissuespecific biological processes and indicates that a specific tissue configuration, i.e., the one that maps a gene to its tissue of maximum variance (thus, the tissue which perhaps most accurately reflects the gene's range of functional activity), may be unique for its constraint on evolutionary rate. The observed constraint of the regulatory genome (with its potential tissue specificity) on evolutionary rate and the evidence for the tissue-anchored model would suggest the importance of the developmental and 
692 physiological processes in which transcription takes place (thus determining gene function in a context-

693 specific way) as a primary driver of protein sequence evolution in humans. Notably, using a reference

694 UK-wide genomic resource, we find that the MaxTissue for a gene significantly predicts the primary

695 affected tissue for developmental disorders associated with the gene, lending substantial empirical

696 support to the tissue-anchored model since developmental disorders are likely to affect fitness and thus 697 evolutionary rate.

We explored several methodological applications of our evolutionary analysis to genomic

699

700

701

702

703

704

705

706

707

708

709

710

711

712

713

714 studies of disease. Gene expression imputation (Gamazon et al., 2015a; Wang et al., 2016) is a powerful approach to mapping disease-associated genes that is now becoming more widely used (Hoffman et al., 2017; Son et al., 2017; Xu et al., 2017, 2018; Zeng, Wang \& Huang, 2017; Gottlieb et al., 2017; Li et al., 2018; Sanchez-Roige et al., 2018; Lamontagne et al., 2018). In this framework, the imputed genetically determined expression in a tissue is tested for its contribution to disease phenotype. An observed correlation with the genetically determined component (unlike the directly measured expression level) proposes a causal direction of effect, as the disease trait is not likely to alter the germline genetic profile. (Of course, definitive identification of causal genes may ultimately require perturbation experiments.) Thus, estimating the genetically determined component of gene expression extends differential expression analysis of the directly measured gene expression. As predicted by our framework, the expression of conserved genes is not as accurately imputed, using local genetic variation, as the expression of fast-evolving genes. Thus, transcriptome-wide association studies, such as implemented in PrediXcan (Gamazon et al., 2015a), in search of disease-associated genes may be substantially enhanced by incorporating the possible determinants of evolutionary rate into the analysis. Since Mendelian disease genes are enriched for conserved genes, the lower imputation quality for these genes implies that their reported effects on complex traits from local genetic variation may be severely

Peer) reviewing PDF | (2020:03:46779:1:2:NEW 16 Jun 2020) 
715 underestimated despite the substantial comorbidity associations between Mendelian and complex

716 disorders that have been uncovered (Blair et al., 2013).

A major methodological implication of our study is the importance of building evolutionary-rateaware models of genetically determined expression for increased statistical power. Fast-evolving genes tend to be highly tissue-specific, suggesting the importance of tissue-specific imputation models for these genes. On the other hand, genes under strong purifying selection, including essential genes, tend to be broadly expressed, suggesting the importance of multi-tissue imputation models that explicitly utilize their tissue-shared expression profile. Current models, which are indifferent to evolutionary rate and its contributing features, are thus likely to be underpowered for a class of genes. constrain protein sequence evolution. We highlight the importance of cross-tissue predictors, whose properties can be more accurately characterized using a more comprehensive tissue collection than previously available and a uniform analysis pipeline. Importantly, we show, using gradient boosted regression, that the feature set explains $62 \%$ of the variability in evolutionary rate and provides additional support to the tissue-anchored model. The tissue-anchored model reinforces the notion that rather than a single mechanism (gene expression) being significant for protein evolution, variation in the conservation of certain developmental programs in which transcriptional dynamics unfolds in space and time may be a primary driver of evolutionary rate. Mechanistically, the regulatory genome may constrain evolutionary rate through its overall effect on the phenome (Nicolae et al., 2010; GTEx constraints on protein or mRNA folding (Drummond et al., 2005; Yang et al., 2012), Mendelian disease 
737 random genes. This observation, coupled with the tissue-anchored model, supports the greater role of

738 gene function than of protection from protein misfolding, in the evolution of these genes.

Our study used species divergence to measure protein evolution and selection, but we have not utilized the polymorphism data within GTEx to examine signatures of more recent selection (Voight et al., 2006; Field et al., 2016). It would be interesting to test whether our conclusions hold at shorter

742 human evolutionary time-scales. Furthermore, the use of additional species (given the availability of dozens of mammalian genomes now) may improve the evolutionary analyses presented here. We also examined mRNA expression, which may not reflect protein-specific regulatory mechanisms.

Incorporation of protein expression, translational efficiency, and codon bias will likely improve our model of protein evolution and provide additional mechanistic insights into disease biology.

\section{Conclusions} framework, assessed their relevance to characterizing human disease-associated genes, and explored methodological implications for transcriptome-wide association studies. We propose a tissue-anchored model for protein evolutionary rate and a combined feature set that explains a large proportion of the variation. The tissue-anchored model provides a transcriptome-based approach to predicting the 


\section{Acknowledgments}

757 We would like to thank Jibril Hirbo for his editorial feedback. E.R.G. is grateful to the President and

758 Fellows of Clare Hall, University of Cambridge for providing a stimulating intellectual home during his

759 Visiting Fellowship.

760

761

\section{References}

762

763

764

765

766

767

768

769

770

771

772

773

774

775

776

777

778

779

Aggarwala V, Voight BF. 2016. An expanded sequence context model broadly explains variability in polymorphism levels across the human genome. Nature Genetics 48:349355. DOI: $10.1038 / \mathrm{ng} .3511$.

Akashi H. 2001. Gene expression and molecular evolution. Current Opinion in Genetics \& Development 11:660-666.

Akashi H. 2003. Translational selection and yeast proteome evolution. Genetics 164:1291-1303.

Ashburner M, Ball CA, Blake JA, Botstein D, Butler H, Cherry JM, Davis AP, Dolinski K, Dwight SS, Eppig JT, Harris MA, Hill DP, Issel-Tarver L, Kasarskis A, Lewis S, Matese JC, Richardson JE, Ringwald M, Rubin GM, Sherlock G. 2000. Gene ontology: tool for the unification of biology. The Gene Ontology Consortium. Nature Genetics 25:25-29. DOI: $10.1038 / 75556$.

Blair DR, Lyttle CS, Mortensen JM, Bearden CF, Jensen AB, Khiabanian H, Melamed R, Rabadan R, Bernstam EV, Brunak S, Jensen LJ, Nicolae D, Shah NH, Grossman RL, Cox NJ, White KP, Rzhetsky A. 2013. A nondegenerate code of deleterious variants in Mendelian loci contributes to complex disease risk. Cell 155:70-80. DOI: 10.1016/j.cell.2013.08.030.

Blake JA, Bult CJ, Kadin JA, Richardson JE, Eppig JT, Mouse Genome Database Group. 2011. The Mouse Genome Database (MGD): premier model organism resource for mammalian 
genomics and genetics. Nucleic Acids Research 39:D842-848. DOI:

781 10.1093/nar/gkq1008.

782

783

784

785

786

787

788

789

790

791

792

793

794

795

796

797

798

799

800

801

802

Chhibber A, French CE, Yee SW, Gamazon ER, Theusch E, Qin X, Webb A, Papp AC, Wang A, Simmons CQ, Konkashbaev A, Chaudhry AS, Mitchel K, Stryke D, Ferrin TE, Weiss ST, Kroetz DL, Sadee W, Nickerson DA, Krauss RM, George AL, Schuetz EG, Medina MW, Cox NJ, Scherer SE, Giacomini KM, Brenner SE. 2017. Transcriptomic variation of pharmacogenes in multiple human tissues and lymphoblastoid cell lines. The Pharmacogenomics Journal 17:137-145. DOI: 10.1038/tpj.2015.93.

Drummond DA, Bloom JD, Adami C, Wilke CO, Arnold FH. 2005. Why highly expressed proteins evolve slowly. Proceedings of the National Academy of Sciences of the United States of America 102:14338-14343. DOI: 10.1073/pnas.0504070102.

Drummond DA, Raval A, Wilke CO. 2006. A single determinant dominates the rate of yeast protein evolution. Molecular Biology and Evolution 23:327-337. DOI: 10.1093/molbev/msj038.

Drummond DA, Wilke CO. 2008. Mistranslation-induced protein misfolding as a dominant constraint on coding-sequence evolution. Cell 134:341-352. DOI: 10.1016/j.cell.2008.05.042.

Duret L, Mouchiroud D. 2000. Determinants of substitution rates in mammalian genes: expression pattern affects selection intensity but not mutation rate. Molecular Biology and Evolution 17:68-74.

Field Y, Boyle EA, Telis N, Gao Z, Gaulton KJ, Golan D, Yengo L, Rocheleau G, Froguel P, McCarthy MI, Pritchard JK. 2016. Detection of human adaptation during the past 2000 years. Science (New York, N.Y.) 354:760-764. DOI: 10.1126/science.aag0776. 
803 Fraser HB, Hirsh AE, Steinmetz LM, Scharfe C, Feldman MW. 2002. Evolutionary rate in the 804 protein interaction network. Science (New York, N.Y.) 296:750-752. DOI:

805

806

807

808

809

810

811

812

813

814

815

816

817

818

819

820

821

822

823

824 10.1126/science. 1068696 .

Gamazon ER, Wheeler HE, Shah KP, Mozaffari SV, Aquino-Michaels K, Carroll RJ, Eyler AE, Denny JC, GTEx Consortium, Nicolae DL, Cox NJ, Im HK. 2015a. A gene-based association method for mapping traits using reference transcriptome data. Nature Genetics 47:1091-1098. DOI: 10.1038/ng.3367.

Gamazon ER, Wheeler HE, Shah KP, Mozaffari SV, Aquino-Michaels K, Carroll RJ, Eyler AE, Denny JC, GTEx Consortium, Nicolae DL, Cox NJ, Im HK. 2015b. A gene-based association method for mapping traits using reference transcriptome data. Nature Genetics 47:1091-1098. DOI: 10.1038/ng.3367.

Gene Ontology Consortium. 2015. Gene Ontology Consortium: going forward. Nucleic Acids Research 43:D1049-1056. DOI: 10.1093/nar/gku1179.

Georgi B, Voight BF, Bućan M. 2013. From mouse to human: evolutionary genomics analysis of human orthologs of essential genes. PLoS genetics 9:e1003484. DOI: 10.1371/journal.pgen.1003484.

Gottlieb A, Daneshjou R, DeGorter M, Bourgeois S, Svensson PJ, Wadelius M, Deloukas P, Montgomery SB, Altman RB. 2017. Cohort-specific imputation of gene expression improves prediction of warfarin dose for African Americans. Genome Medicine 9:98. DOI: $10.1186 / \mathrm{s} 13073-017-0495-0$.

GTEx Consortium. 2013. The Genotype-Tissue Expression (GTEx) project. Nature Genetics 45:580-585. DOI: $10.1038 / n g .2653$. 
825 GTEx Consortium. 2015. Human genomics. The Genotype-Tissue Expression (GTEx) pilot 826 analysis: multitissue gene regulation in humans. Science (New York, N.Y.) 348:648-660.

827

828

829

830

831

832

833

834

835

836

837

838

839

840

841

842

843

844

845

846

847 DOI: $10.1126 /$ science. 1262110.

$\mathrm{Gu} \mathrm{X}, \mathrm{Su} \mathrm{Z} .2007$. Tissue-driven hypothesis of genomic evolution and sequence-expression correlations. Proceedings of the National Academy of Sciences of the United States of America 104:2779-2784. DOI: 10.1073/pnas.0610797104.

Gusev A, Ko A, Shi H, Bhatia G, Chung W, Penninx BWJH, Jansen R, de Geus EJC, Boomsma DI, Wright FA, Sullivan PF, Nikkola E, Alvarez M, Civelek M, Lusis AJ, Lehtimäki T, Raitoharju E, Kähönen M, Seppälä I, Raitakari OT, Kuusisto J, Laakso M, Price AL, Pajukanta P, Pasaniuc B. 2016. Integrative approaches for large-scale transcriptome-wide association studies. Nature Genetics 48:245-252. DOI: 10.1038/ng.3506.

Hochstrasser M. 2009. Origin and function of ubiquitin-like proteins. Nature 458:422-429. DOI: 10.1038/nature07958.

Hoffman JD, Graff RE, Emami NC, Tai CG, Passarelli MN, Hu D, Huntsman S, Hadley D, Leong L, Majumdar A, Zaitlen N, Ziv E, Witte JS. 2017. Cis-eQTL-based trans-ethnic meta-analysis reveals novel genes associated with breast cancer risk. PLoS genetics 13:e1006690. DOI: 10.1371/journal.pgen.1006690.

Huang DW, Sherman BT, Lempicki RA. 2009. Bioinformatics enrichment tools: paths toward the comprehensive functional analysis of large gene lists. Nucleic Acids Research 37:113. DOI: $10.1093 /$ nar/gkn923.

Hudson CM, Conant GC. 2011. Expression level, cellular compartment and metabolic network position all influence the average selective constraint on mammalian enzymes. $B M C$ evolutionary biology 11:89. DOI: 10.1186/1471-2148-11-89. 
848 Jordan IK, Rogozin IB, Wolf YI, Koonin EV. 2002. Essential genes are more evolutionarily

849 conserved than are nonessential genes in bacteria. Genome Research 12:962-968. DOI:

$850 \quad$ 10.1101/gr.87702. Article published online before print in May 2002.

851 Kimura M. 1990. The neutral theory of molecular evolution. Cambridge: Cambridge Univ. Press.

852 King MC, Wilson AC. 1975. Evolution at two levels in humans and chimpanzees. Science (New $853 \quad$ York, N.Y.) 188:107-116.

854 Kryuchkova-Mostacci N, Robinson-Rechavi M. 2015. Tissue-Specific Evolution of Protein 855 Coding Genes in Human and Mouse. PloS One 10:e0131673. DOI:

$856 \quad$ 10.1371/journal.pone.0131673.

857 Kryuchkova-Mostacci N, Robinson-Rechavi M. 2016. A benchmark of gene expression tissue858 specificity metrics. Briefings in Bioinformatics. DOI: 10.1093/bib/bbw008.

859 Lamontagne M, Bérubé J-C, Obeidat M, Cho MH, Hobbs BD, Sakornsakolpat P, de Jong K, 860 Boezen HM, International COPD Genetics Consortium, Nickle D, Hao K, Timens W, 861 van den Berge M, Joubert P, Laviolette M, Sin DD, Paré PD, Bossé Y. 2018. Leveraging 862 lung tissue transcriptome to uncover candidate causal genes in COPD genetic 863 associations. Human Molecular Genetics. DOI: 10.1093/hmg/ddy091.

864 Lappalainen T, Sammeth M, Friedländer MR, ’t Hoen PAC, Monlong J, Rivas MA, Gonzàlez-

865 Porta M, Kurbatova N, Griebel T, Ferreira PG, Barann M, Wieland T, Greger L, van 866 Iterson M, Almlöf J, Ribeca P, Pulyakhina I, Esser D, Giger T, Tikhonov A, Sultan M, Bertier G, MacArthur DG, Lek M, Lizano E, Buermans HPJ, Padioleau I, Schwarzmayr T, Karlberg O, Ongen H, Kilpinen H, Beltran S, Gut M, Kahlem K, Amstislavskiy V, Stegle O, Pirinen M, Montgomery SB, Donnelly P, McCarthy MI, Flicek P, Strom TM, Geuvadis Consortium, Lehrach H, Schreiber S, Sudbrak R, Carracedo A, Antonarakis 
871

872

873

874

875

876

877

878

879

880

881

882

883

884

885

886

887

888

889

890

891

892

893

SE, Häsler R, Syvänen A-C, van Ommen G-J, Brazma A, Meitinger T, Rosenstiel P, Guigó R, Gut IG, Estivill X, Dermitzakis ET. 2013. Transcriptome and genome sequencing uncovers functional variation in humans. Nature 501:506-511. DOI: 10.1038/nature12531.

Larracuente AM, Sackton TB, Greenberg AJ, Wong A, Singh ND, Sturgill D, Zhang Y, Oliver B, Clark AG. 2008. Evolution of protein-coding genes in Drosophila. Trends in genetics: TIG 24:114-123. DOI: 10.1016/j.tig.2007.12.001.

Lemos B, Meiklejohn CD, Hartl DL. 2004. Regulatory evolution across the protein interaction network. Nature Genetics 36:1059-1060. DOI: 10.1038/ng1427.

Li B, Verma SS, Veturi YC, Verma A, Bradford Y, Haas DW, Ritchie MD. 2018. Evaluation of PrediXcan for prioritizing GWAS associations and predicting gene expression. Pacific Symposium on Biocomputing. Pacific Symposium on Biocomputing 23:448-459.

Liao B-Y, Scott NM, Zhang J. 2006. Impacts of gene essentiality, expression pattern, and gene compactness on the evolutionary rate of mammalian proteins. Molecular Biology and Evolution 23:2072-2080. DOI: 10.1093/molbev/ms1076.

Liu K. 1988. Measurement error and its impact on partial correlation and multiple linear regression analyses. American Journal of Epidemiology 127:864-874.

MacArthur DG, Balasubramanian S, Frankish A, Huang N, Morris J, Walter K, Jostins L, Habegger L, Pickrell JK, Montgomery SB, Albers CA, Zhang ZD, Conrad DF, Lunter G, Zheng H, Ayub Q, DePristo MA, Banks E, Hu M, Handsaker RE, Rosenfeld JA, Fromer M, Jin M, Mu XJ, Khurana E, Ye K, Kay M, Saunders GI, Suner M-M, Hunt T, Barnes IHA, Amid C, Carvalho-Silva DR, Bignell AH, Snow C, Yngvadottir B, Bumpstead S, Cooper DN, Xue Y, Romero IG, 1000 Genomes Project Consortium, Wang J, Li Y, 
894

895

896

897

898

899

900

901

902

903

904

905

906

907

908

909

910

911

912

913

914

915

916

Gibbs RA, McCarroll SA, Dermitzakis ET, Pritchard JK, Barrett JC, Harrow J, Hurles ME, Gerstein MB, Tyler-Smith C. 2012. A systematic survey of loss-of-function variants in human protein-coding genes. Science (New York, N.Y.) 335:823-828. DOI:

10.1126/science. 1215040 .

Martin HC, Jones WD, McIntyre R, Sanchez-Andrade G, Sanderson M, Stephenson JD, Jones CP, Handsaker J, Gallone G, Bruntraeger M, McRae JF, Prigmore E, Short P, Niemi M, Kaplanis J, Radford EJ, Akawi N, Balasubramanian M, Dean J, Horton R, Hulbert A, Johnson DS, Johnson K, Kumar D, Lynch SA, Mehta SG, Morton J, Parker MJ, Splitt M, Turnpenny PD, Vasudevan PC, Wright M, Bassett A, Gerety SS, Wright CF, FitzPatrick DR, Firth HV, Hurles ME, Barrett JC, Deciphering Developmental Disorders Study. 2018. Quantifying the contribution of recessive coding variation to developmental disorders. Science (New York, N.Y.) 362:1161-1164. DOI: 10.1126/science.aar6731.

Moyers BA, Zhang J. 2017. Further Simulations and Analyses Demonstrate Open Problems of Phylostratigraphy. Genome Biology and Evolution 9:1519-1527. DOI: $10.1093 /$ gbe/evx 109.

Nicolae DL, Gamazon E, Zhang W, Duan S, Dolan ME, Cox NJ. 2010. Trait-associated SNPs are more likely to be eQTLs: annotation to enhance discovery from GWAS. PLoS genetics 6:e1000888. DOI: 10.1371/journal.pgen.1000888.

Pál C, Papp B, Hurst LD. 2001. Highly expressed genes in yeast evolve slowly. Genetics 158:927-931.

Park SG, Choi SS. 2010. Expression breadth and expression abundance behave differently in correlations with evolutionary rates. BMC evolutionary biology 10:241. DOI: $10.1186 / 1471-2148-10-241$ 
917 Park J, Xu K, Park T, Yi SV. 2012. What are the determinants of gene expression levels and

918 breadths in the human genome? Human Molecular Genetics 21:46-56. DOI:

$919 \quad 10.1093 / \mathrm{hmg} / \mathrm{ddr} 436$.

920 Pickrell JK, Marioni JC, Pai AA, Degner JF, Engelhardt BE, Nkadori E, Veyrieras J-B, Stephens

921 M, Gilad Y, Pritchard JK. 2010. Understanding mechanisms underlying human gene

922 expression variation with RNA sequencing. Nature 464:768-772. DOI:

$923 \quad 10.1038 /$ nature08872.

924 Plotkin JB, Kudla G. 2011. Synonymous but not the same: the causes and consequences of codon 925 bias. Nature Reviews. Genetics 12:32-42. DOI: 10.1038/nrg2899.

926 Quax TEF, Claassens NJ, Söll D, van der Oost J. 2015. Codon Bias as a Means to Fine-Tune

927 Gene Expression. Molecular Cell 59:149-161. DOI: 10.1016/j.molcel.2015.05.035.

928 Rocha EPC, Danchin A. 2004. An analysis of determinants of amino acids substitution rates in 929 bacterial proteins. Molecular Biology and Evolution 21:108-116. DOI: 10.1093/molbev/msh004.

Roff D. 2000. The evolution of the G matrix: selection or drift? Heredity 84 ( Pt 2):135-142.

932 Sanchez-Roige S, Fontanillas P, Elson SL, Pandit A, Schmidt EM, Foerster JR, Abecasis GR, Gray JC, de Wit H, Davis LK, MacKillop J, Palmer AA, 23andMe Research Team. 2018. Genome-wide association study of delay discounting in 23,217 adult research participants of European ancestry. Nature Neuroscience 21:16-18. DOI: 10.1038/s41593-017-0032-x. microRNAs. Proceedings of the National Academy of Sciences of the United States of America 108:15942-15947. DOI: 10.1073/pnas.1110098108. 
940 Smedley D, Haider S, Durinck S, Pandini L, Provero P, Allen J, Arnaiz O, Awedh MH, Baldock

941 R, Barbiera G, Bardou P, Beck T, Blake A, Bonierbale M, Brookes AJ, Bucci G, Buetti I,

942 Burge S, Cabau C, Carlson JW, Chelala C, Chrysostomou C, Cittaro D, Collin O,

943 Cordova R, Cutts RJ, Dassi E, Di Genova A, Djari A, Esposito A, Estrella H, Eyras E,

944 Fernandez-Banet J, Forbes S, Free RC, Fujisawa T, Gadaleta E, Garcia-Manteiga JM,

945 Goodstein D, Gray K, Guerra-Assunção JA, Haggarty B, Han D-J, Han BW, Harris T,

946 Harshbarger J, Hastings RK, Hayes RD, Hoede C, Hu S, Hu Z-L, Hutchins L, Kan Z,

947

948

949

950

951

952

953

954

955

Kawaji H, Keliet A, Kerhornou A, Kim S, Kinsella R, Klopp C, Kong L, Lawson D,

Lazarevic D, Lee J-H, Letellier T, Li C-Y, Lio P, Liu C-J, Luo J, Maass A, Mariette J,

Maurel T, Merella S, Mohamed AM, Moreews F, Nabihoudine I, Ndegwa N, Noirot C,

Perez-Llamas C, Primig M, Quattrone A, Quesneville H, Rambaldi D, Reecy J, Riba M, Rosanoff S, Saddiq AA, Salas E, Sallou O, Shepherd R, Simon R, Sperling L, Spooner

W, Staines DM, Steinbach D, Stone K, Stupka E, Teague JW, Dayem Ullah AZ, Wang J, Ware D, Wong-Erasmus M, Youens-Clark K, Zadissa A, Zhang S-J, Kasprzyk A. 2015.

Son H-Y, Hwangbo Y, Yoo S-K, Im S-W, Yang SD, Kwak S-J, Park MS, Kwak SH, Cho SW,

The BioMart community portal: an innovative alternative to large, centralized data repositories. Nucleic Acids Research 43:W589-598. DOI: 10.1093/nar/gkv350.

958 Ryu JS, Kim J, Jung Y-S, Kim TH, Kim S-J, Lee KE, Park DJ, Cho NH, Sung J, Seo J-S, Lee EK, Park YJ, Kim J-I. 2017. Genome-wide association and expression quantitative trait loci studies identify multiple susceptibility loci for thyroid cancer. Nature Communications 8:15966. DOI: 10.1038/ncomms15966. 
961 Stegle O, Parts L, Piipari M, Winn J, Durbin R. 2012. Using probabilistic estimation of

962

963

964

965

966

967

968

969

970

971

972

973

974

975

976

977

978

979

980

981

982 expression residuals (PEER) to obtain increased power and interpretability of gene expression analyses. Nature Protocols 7:500-507. DOI: 10.1038/nprot.2011.457.

Stone M. An asymptotic equivalence of choice of model by cross-validation and Akaike's criterion. Journal of the Royal Statistical Society: Series B (Methodological) 39:44-47.

Szklarczyk D, Franceschini A, Wyder S, Forslund K, Heller D, Huerta-Cepas J, Simonovic M, Roth A, Santos A, Tsafou KP, Kuhn M, Bork P, Jensen LJ, von Mering C. 2015. STRING v10: protein-protein interaction networks, integrated over the tree of life. Nucleic Acids Research 43:D447-452. DOI: 10.1093/nar/gku1003.

Tuller T, Kupiec M, Ruppin E. 2008. Evolutionary rate and gene expression across different brain regions. Genome Biology 9:R142. DOI: 10.1186/gb-2008-9-9-r142.

Voight BF, Kudaravalli S, Wen X, Pritchard JK. 2006. A map of recent positive selection in the human genome. PLoS biology 4:e72. DOI: 10.1371/journal.pbio.0040072.

Wagner A. 2005. Energy Constraints on the Evolution of Gene Expression. Molecular Biology and Evolution 22:1365-1374. DOI: 10.1093/molbev/msi126.

Wall DP, Hirsh AE, Fraser HB, Kumm J, Giaever G, Eisen MB, Feldman MW. 2005. Functional genomic analysis of the rates of protein evolution. Proceedings of the National Academy of Sciences of the United States of America 102:5483-5488. DOI:

10.1073/pnas.0501761102.

Wang J, Gamazon ER, Pierce BL, Stranger BE, Im HK, Gibbons RD, Cox NJ, Nicolae DL, Chen LS. 2016. Imputing Gene Expression in Uncollected Tissues Within and Beyond GTEx. American Journal of Human Genetics 98:697-708. DOI: 10.1016/j.ajhg.2016.02.020. 
983 Xu H, Dorn Ii GW, Shetty A, Parihar A, Dave T, Robinson SW, Gottlieb SS, Donahue MP,

984 Tomaselli GF, Kraus WE, Mitchell BD, Liggett SB. 2018. A Genome-Wide Association

985 Study of Idiopathic Dilated Cardiomyopathy in African Americans. Journal of

986 Personalized Medicine 8. DOI: 10.3390/jpm8010011.

987 Xu Z, Wu C, Wei P, Pan W. 2017. A Powerful Framework for Integrating eQTL and GWAS

988 Summary Data. Genetics 207:893-902. DOI: 10.1534/genetics.117.300270.

989 Yanai I, Benjamin H, Shmoish M, Chalifa-Caspi V, Shklar M, Ophir R, Bar-Even A, Horn-

990

991

992

Saban S, Safran M, Domany E, Lancet D, Shmueli O. 2005. Genome-wide midrange transcription profiles reveal expression level relationships in human tissue specification.

993

Yang J, Benyamin B, McEvoy BP, Gordon S, Henders AK, Nyholt DR, Madden PA, Heath AC,

994 Martin NG, Montgomery GW, Goddard ME, Visscher PM. 2010. Common SNPs explain

995

996 a large proportion of the heritability for human height. Nature Genetics 42:565-569.

997

Yang J, Lee SH, Goddard ME, Visscher PM. 2011. GCTA: a tool for genome-wide complex trait 998 analysis. American Journal of Human Genetics 88:76-82. DOI:

999 10.1016/j.ajhg.2010.11.011.

Yang J-R, Liao B-Y, Zhuang S-M, Zhang J. 2012. Protein misinteraction avoidance causes 1001 highly expressed proteins to evolve slowly. Proceedings of the National Academy of Sciences of the United States of America 109:E831-840. DOI: 10.1073/pnas.1117408109.

Yang J-R, Zhuang S-M, Zhang J. 2010. Impact of translational error-induced and error-free misfolding on the rate of protein evolution. Molecular Systems Biology 6:421. DOI: $10.1038 / \mathrm{msb} .2010 .78$ 
1006 Zeng P, Wang T, Huang S. 2017. Cis-SNPs Set Testing and PrediXcan Analysis for Gene 1007 Expression Data using Linear Mixed Models. Scientific Reports 7:15237. DOI:

1008 10.1038/s41598-017-15055-8.

1009 Zhang L, Li W-H. 2004. Mammalian housekeeping genes evolve more slowly than tissue-

1010 specific genes. Molecular Biology and Evolution 21:236-239. DOI:

1011 10.1093/molbev/msh010.

1012 Zhang J, Yang J-R. 2015. Determinants of the rate of protein sequence evolution. Nature 1013 Reviews. Genetics 16:409-420. DOI: 10.1038/nrg3950.

1014 Zhou Z, Dang Y, Zhou M, Li L, Yu C-H, Fu J, Chen S, Liu Y. 2016. Codon usage is an 1015

1016

1017 important determinant of gene expression levels largely through its effects on transcription. Proceedings of the National Academy of Sciences of the United States of

1018

1019 


\section{Figure 1}

Correlation of Measures of Protein Sequence Evolution and Expression Features Per Tissue.

A. Heatmap of (Spearman) correlation of evolutionary rate, nonsynonymous substitution rate, and synonymous substitution rate with expression features. Expression (level or variability) constraints on evolutionary rate and synonymous substitution rate vary widely by tissue and show intertissue correlation. B. Concordance between the human-mouse and human-chimp comparisons. The comparisons between the two divergence datasets are shown for the correlation of $d N, d N / d S$, and dS with mean and ratio of variance to mean. $\mathbf{C}$. Robustness of results from technical confounding. PEER factors were used to quantify hidden and technical confounders. The correlation between expression variance and evolutionary rate is highly correlated across tissues between pre-PEER and post-PEER analysis. D. Comparison of human-mouse evolutionary rate and $\log _{2}$ transformed expression level in skeletal muscle. A smooth curve was fitted through the scatter plot with consistent results as the correlation analysis. The orange line denotes fit from least squares regression, red line from LOESS, and blue from Generalized Additive Model. 
A

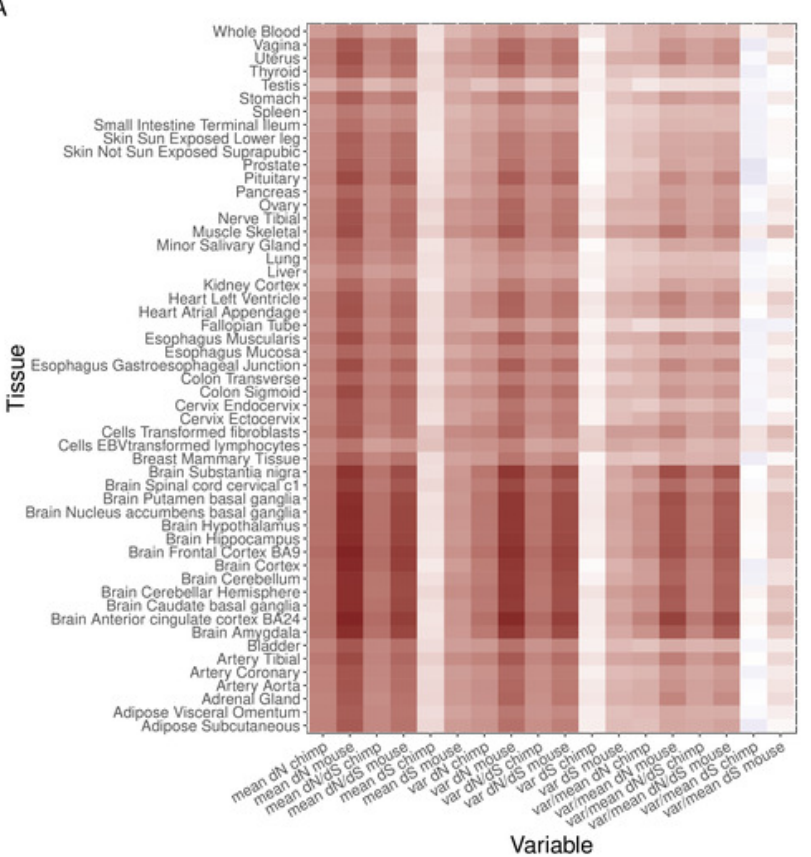

$\mathrm{B}$ 르

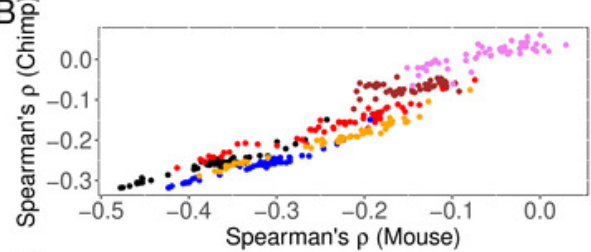

Comparison

- dN mean

- dN variance/mean

dN/dS mean

dN/dS variance/mean

dS mean

dS variance/mean
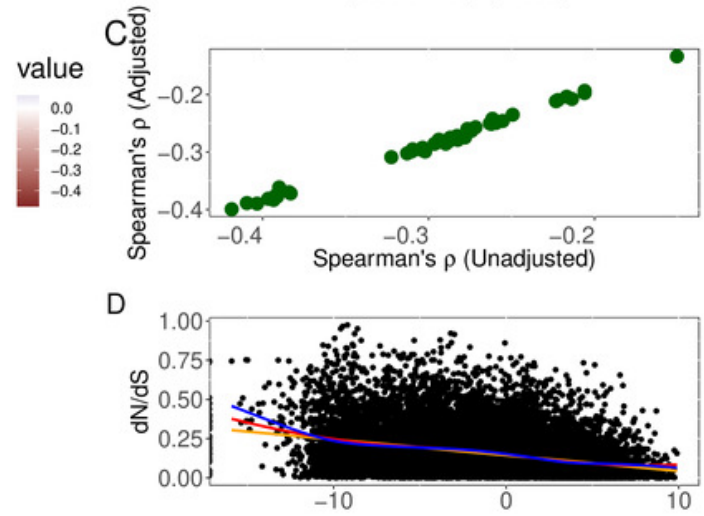

$\log ($ Expression $)$ 


\section{Figure 2}

MaxTissue and the Regulatory Genome as Predictors of Evolutionary Rate.

A. Biological and developmental processes implicated by MaxTissues. MaxTissue is significantly associated with evolutionary rate $\left(p=5.55 \times 10^{-284}\right)$. The top 100 genes with the highest MaxVariance within 3 chosen MaxTissues - cortex, liver, and testis - are enriched for non-overlapping functional annotations. All functional categories shown here satisfy

Benjamini-Hochberg FDR $<0.10$ (raw p-values range from 0.002 to $6.55 \times 10^{-46}$ ) and are in the top 25 in each MaxTissue. In cortex, dendrite, cell junction, and neuron projections are among the functional annotations implicated. In liver: blood microparticle, complement and coagulation cascades, and metabolism of xenobiotics by cytochrome P450. In testis: spermatogenesis, chromosome condensation, and multicellular organism development. The non-overlapping enriched functional annotations and the significant difference in evolutionary rate between MaxTissues suggest the importance of MaxTissue-specific biological and developmental processes in constraining evolutionary rate. B. Estimated tissue specificity of a gene expression trait as a function of number of tissues ( $\mathrm{x}$-axis). Less comprehensive tissue catalogs can have substantial variability ( $y$-axis) in the estimated tissue specificity of gene expression. For a given number of tissues, we randomly selected tissues from the 44 to generate a tissue catalog. We calculated the tissue specificity (tau) of each gene within the tissue catalog. The $y$-axis is the standard deviation of tau across 100 replicates. C. Regulatory genome and protein sequence divergence. The comparison of local genetic control (measured in DGN whole blood transcriptome) between conserved genes and fast-evolving genes is shown. The first, median, and third quartile are displayed as horizontal lines within the two shaded regions. Conserved genes (defined here as the bottom $25 \%$ of the distribution of $d N / d S$ ) have significantly lower (Mann-Whitney $U p=1.2 \times 10^{-17}$ ) cis 
heritability than fast-evolving genes (defined as the top $25 \%$ of the distribution of $\mathrm{dN} / \mathrm{dS}$ ).

The median cis heritability for all genes is 0.058 (significantly higher, $p=9.61 \times 10^{-11}$, than for conserved genes). Degree of local genetic control of gene expression is therefore a predictor of evidence for either purifying selection or positive selection.

A

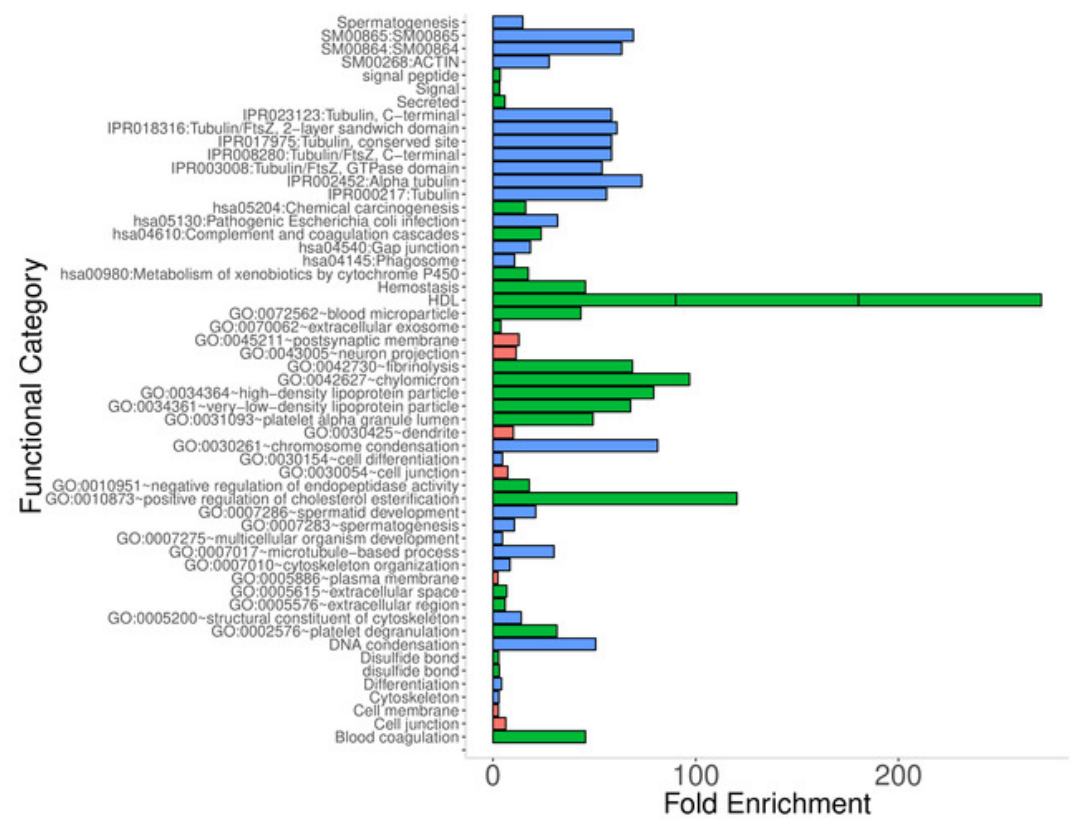

B

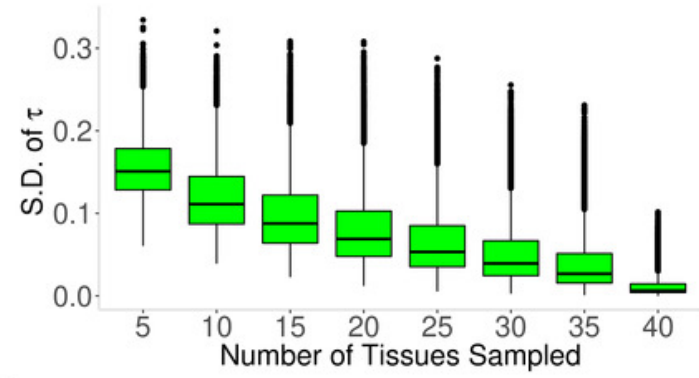

C

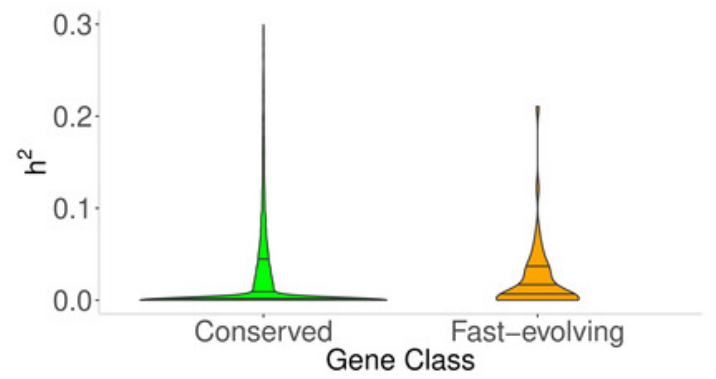




\section{Figure 3 (on next page)}

\section{Joint Analysis of Possible Determinants of Evolutionary Rate.}

A. The pairwise (Spearman) correlation values for all features. Expression breadth is given by the tau statistic with smaller tau indicating wider expression breadth. Pairwise correlation was restricted to proteincoding genes (even though some correlations could be calculated with non-coding genes) because of our focus on protein evolutionary rate. B-J. Comparison of feature importance scores. MaxVariance shows the highest feature importance score using a range of model parameters for the number of trees $(500 ; 1,000$; $10,000)$ and the interaction depth $(1,4,5)$ in gradient boosting. In particular, MaxVariance shows a stronger influence on predicting evolutionary rate than the other cross-tissue features: maximum expression level and expression breadth. 
A

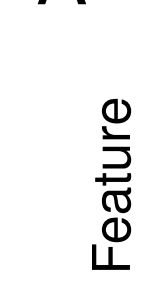

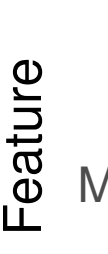

PeerJ

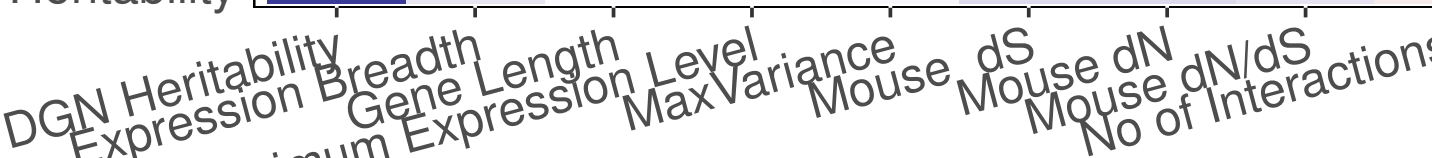

Feature

500 Trees

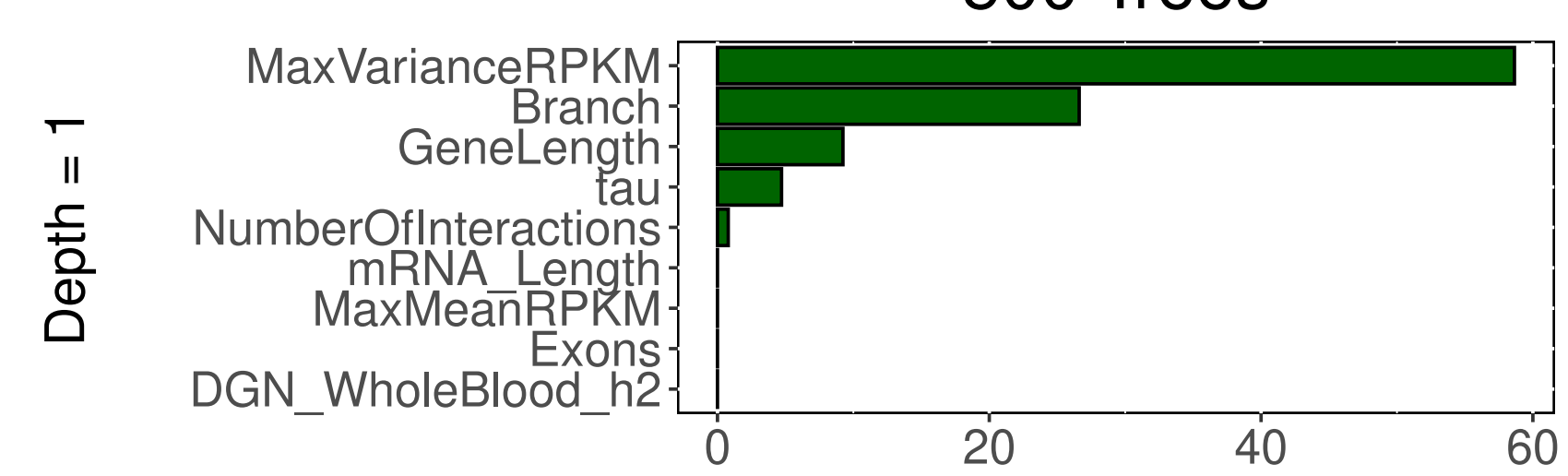

E

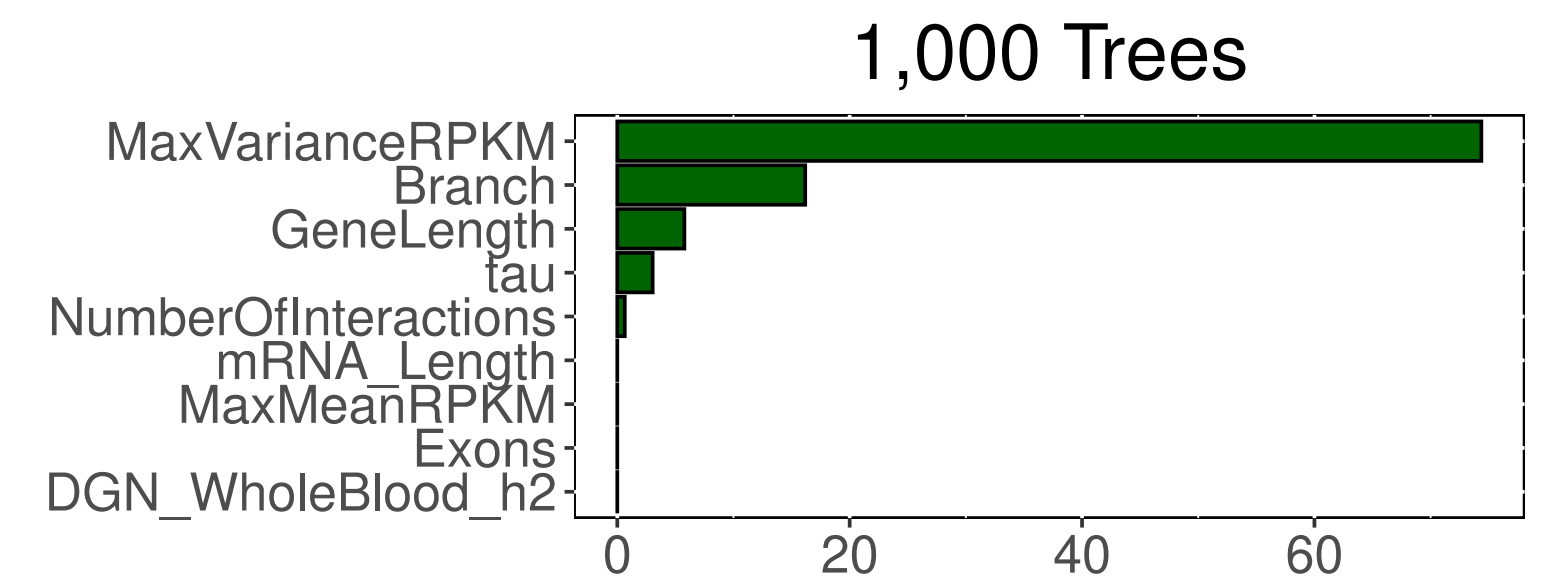

60
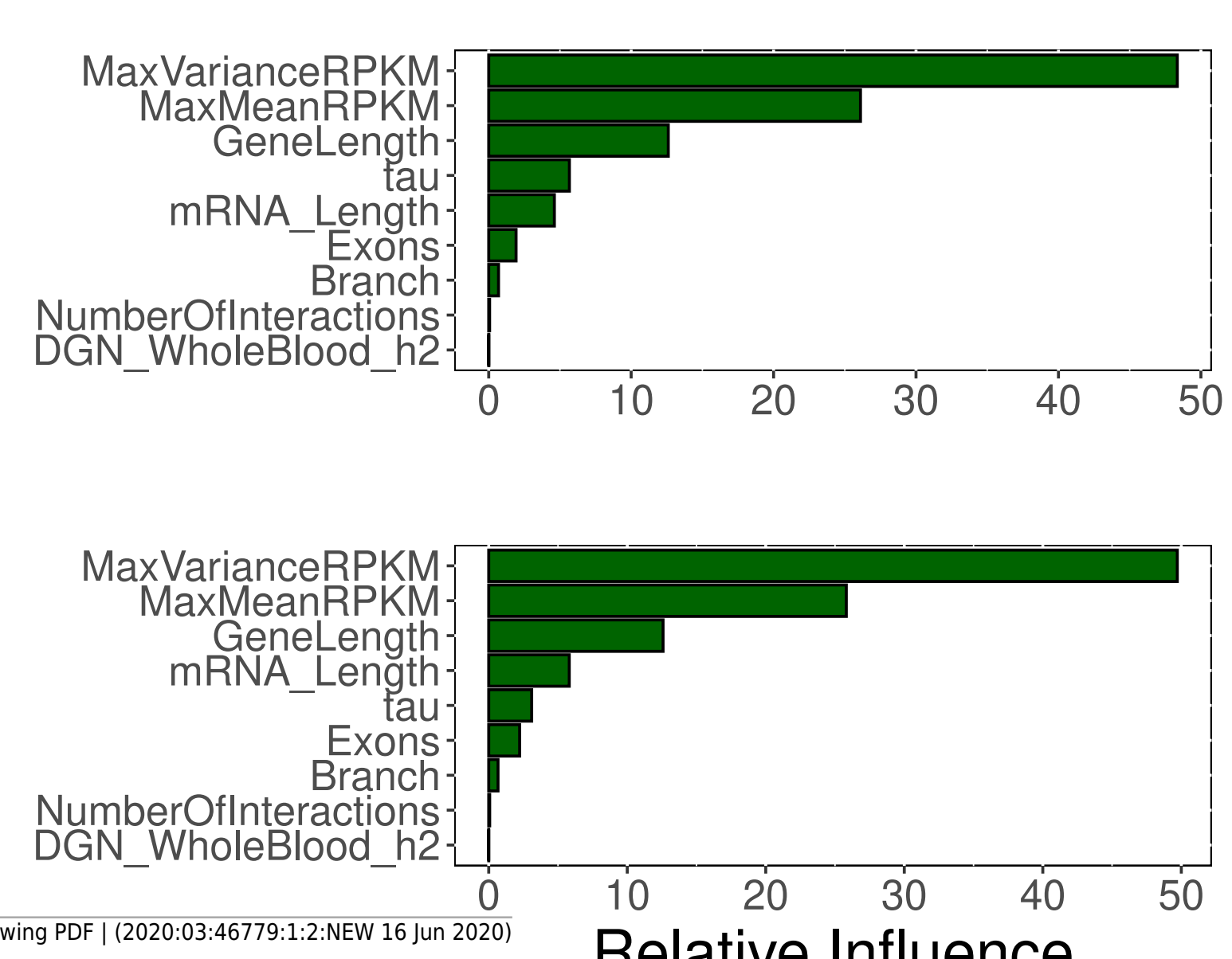

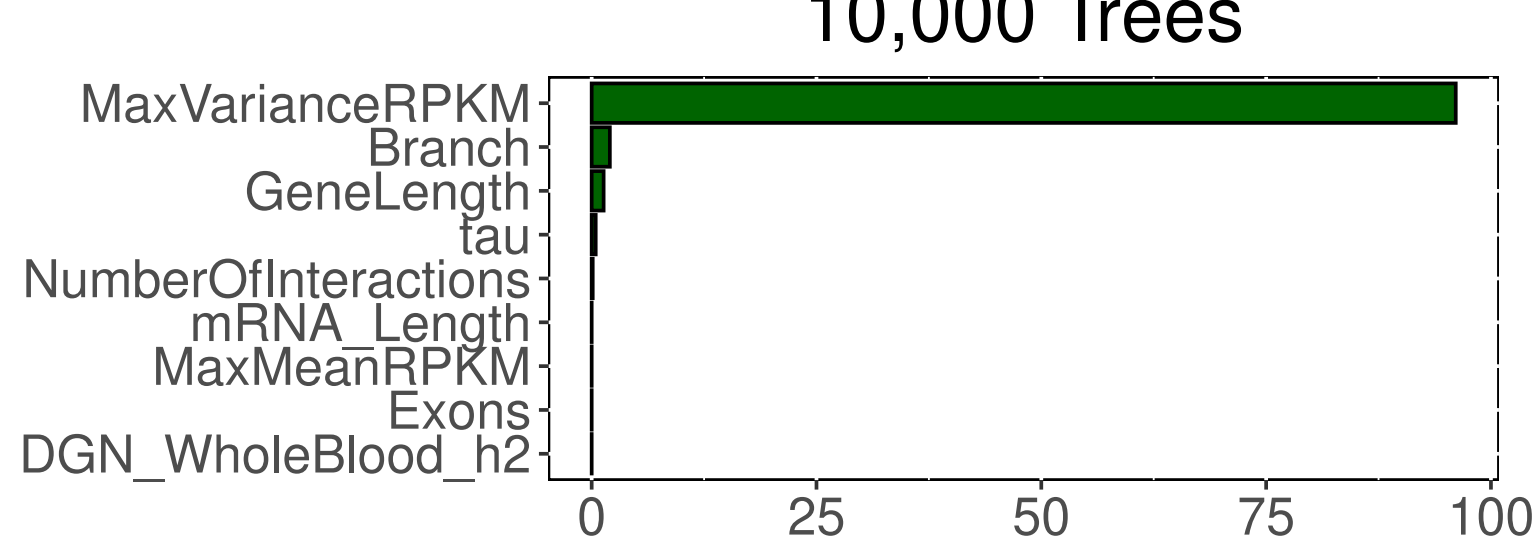

G
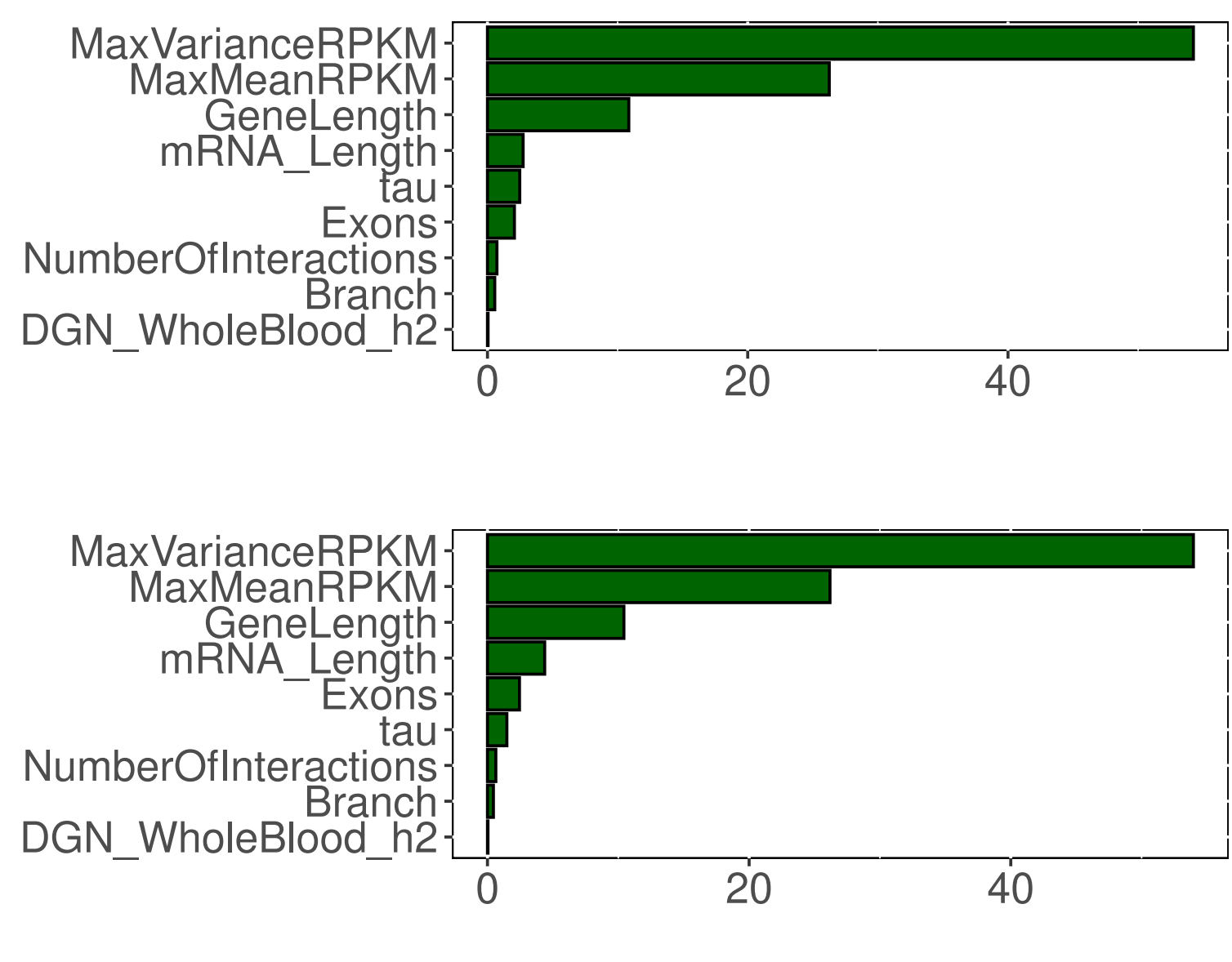

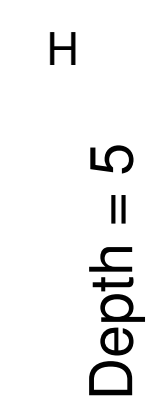

Branch
NumberOfInteractions

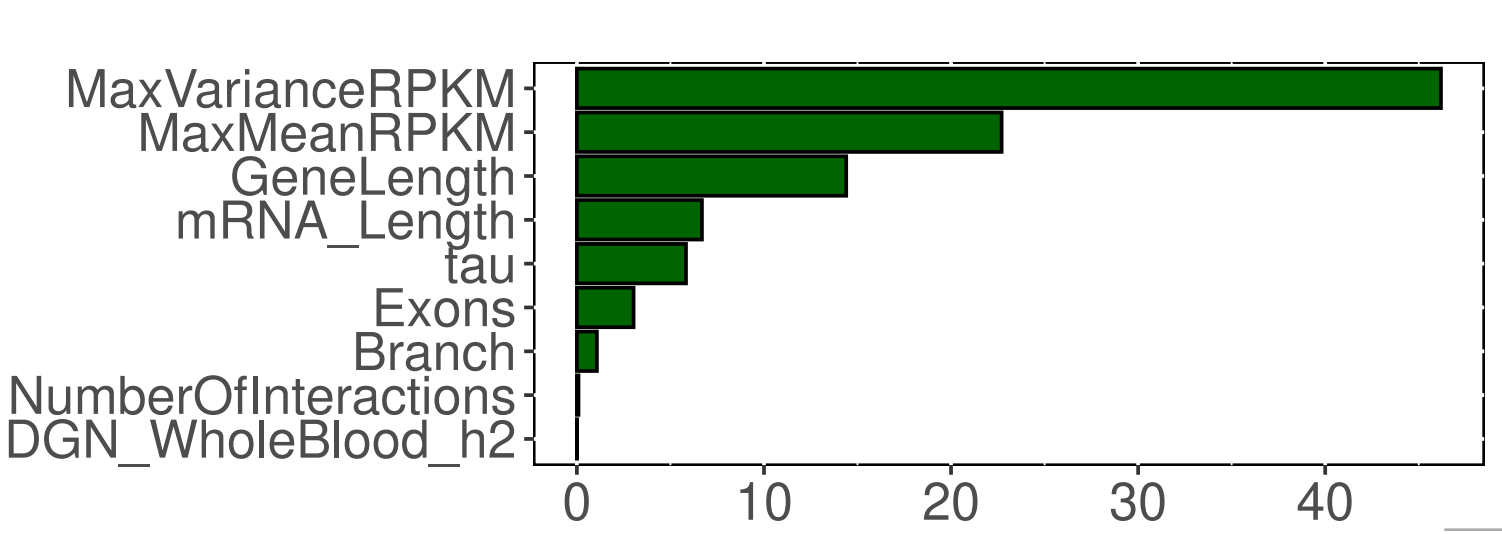




\section{Figure 4}

Model of Evolutionary Rate.

A. Variance explained by model. The joint model from gradient boosting explains an average of $62 \%$ of the variability in evolutionary rate from out-of-sample prediction. The distribution is from 100 cross validation analyses. B. Variability versus level. MaxVariance (right histogram) consistently shows a higher relative importance than maximum expression level (left histogram) in determining evolutionary rate using 100 replicates from gradient boosting. We therefore used MaxVariance to define MaxTissue, a key element of the tissue-anchored model of evolutionary rate. C. Interaction of MaxVariance and function. Mendelian disease genes and essential genes show substantially lower MaxVariance effect (empirical $p<$ 0.001 ), in terms of variance explained, on evolutionary rate than the full set of proteins, indicating that MaxVariance and gene function may interact to constrain evolutionary rate.

A

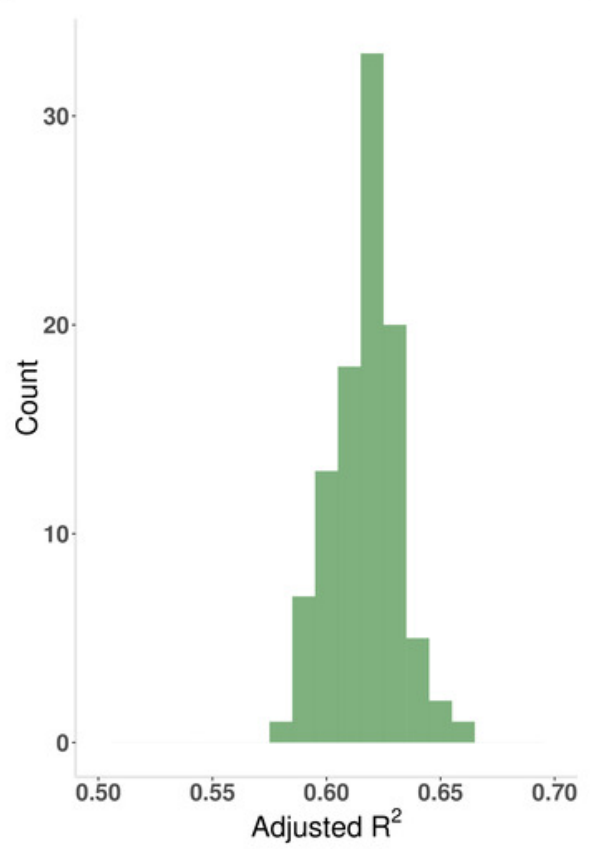

B

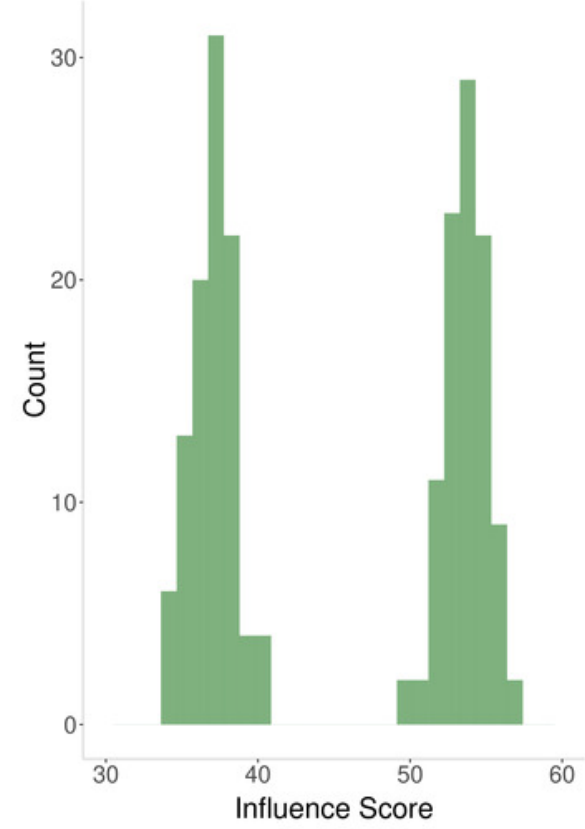

C
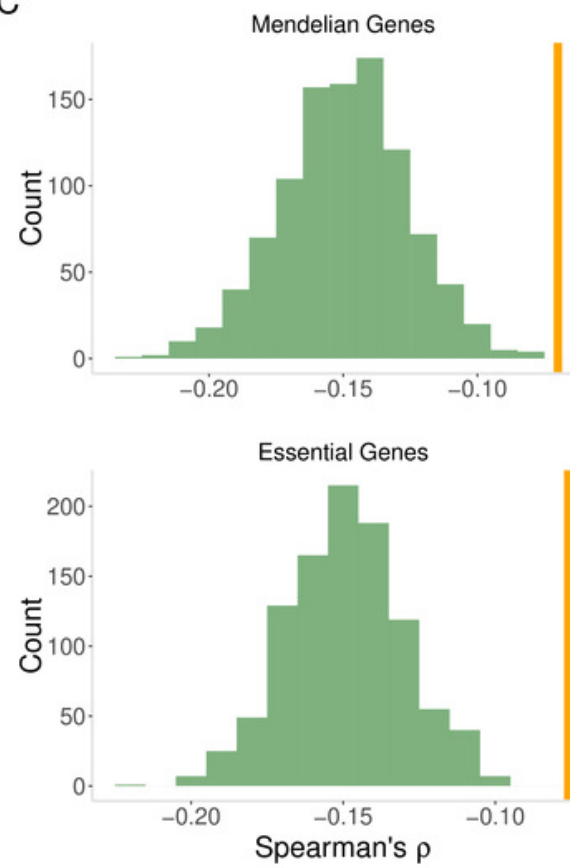


\section{Figure 5}

Correlation between Evolutionary Rate and Out-of-sample Imputation $R^{2}$ for Gene Expression.

A. The out-of-sample $R^{2}$ assumes local predictors and is derived from 10 -fold cross validation within the PrediXcan framework applied to GTEx data. For a wide range of tissues, out-ofsample imputation accuracy is significantly correlated with evolutionary rate. In particular, this suggests that transcriptome-wide association analyses using local predictors in a range of tissues have significantly less power to detect disease associations for conserved genes than for fast-evolving genes. Orange line indicates Bonferroni threshold for the number of tissues tested. B. Conserved genes have significantly lower statistical power than fastevolving genes (median power 0.16 versus 0.41 , Mann-Whitney $U$ test $p=2.2 \times 10^{-6}$ ). Furthermore, a smaller proportion of conserved genes ( 0.32 versus 0.41$)$ would have at least $80 \%$ power. Here skeletal muscle was used.

A

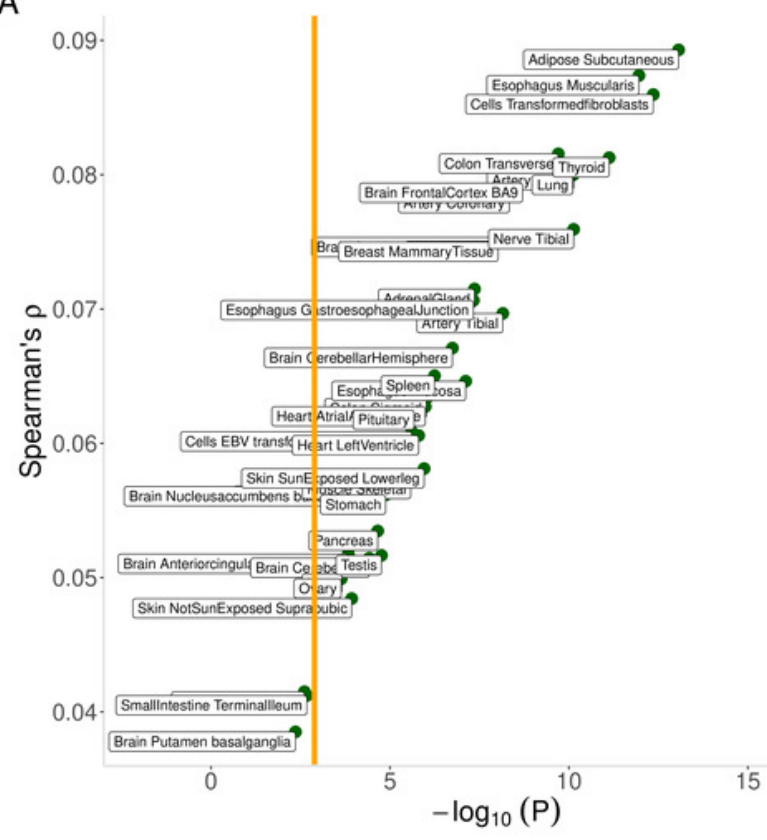

B

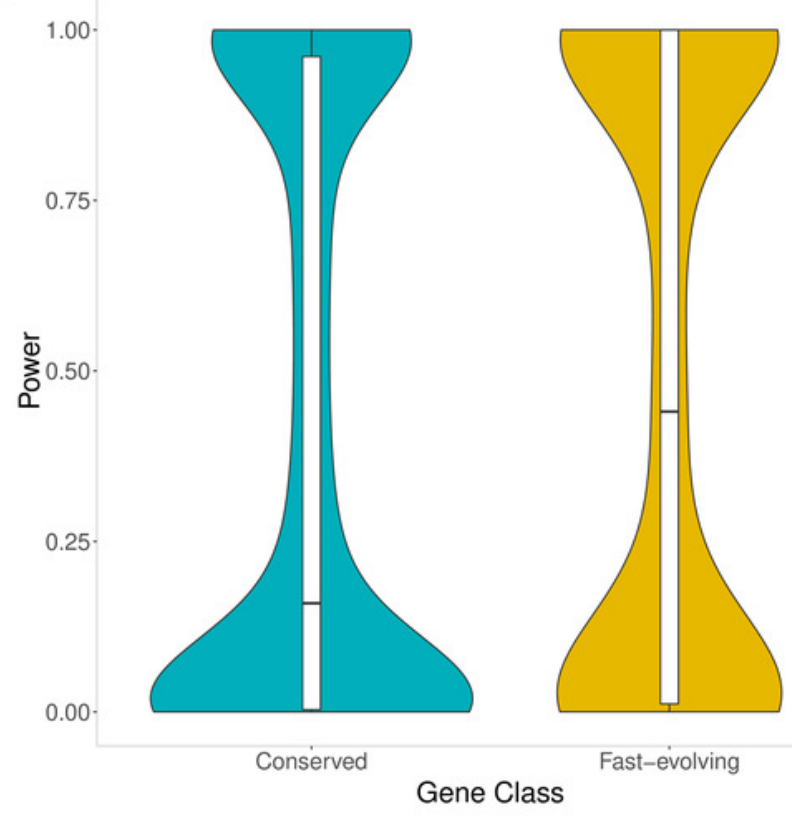

